\title{
TOPOLOGY AND SOBOLEV SPACES
}

\author{
Haim Brezis, ${ }^{(1)},(2)$, AND YANYAN LI, ${ }^{(2)}$
}

\section{SECTION 0. InTRODUCTION}

Let $M$ and $N$ be compact ${ }^{1}$ connected oriented smooth Riemannian manifolds with or without boundary. Throughout the paper we assume that $\operatorname{dim} M \geq 2$ but $\operatorname{dim} N$ could possibly be one, for example $N=\mathbb{S}^{1}$ is of interest. Our functional framework is the Sobolev space $W^{1, p}(M, N)$ which is defined by considering $N$ as smoothly embedded in some Euclidean space $\mathbb{R}^{K}$ and then

$$
W^{1, p}(M, N)=\left\{u \in W^{1, p}\left(M, \mathbb{R}^{K}\right) ; u(x) \in N \text { a.e. }\right\},
$$

with $1 \leq p<\infty . W^{1, p}(M, N)$ is equipped with the standard metric $d(u, v)=\|u-v\|_{W^{1, p}}$. Our main concern is to determine whether or not $W^{1, p}(M, N)$ is path-connected and if not what can be said about its path-connected components, i.e. its $W^{1, p}$-homotopy classes. We say that $u$ and $v$ are $W^{1, p}$-homotopic if there is a path $u^{t} \in C\left([0,1], W^{1, p}(M, N)\right)$ such that $u^{0}=u$ and $u^{1}=v$. We denote by $\sim_{p}$ the corresponding equivalence relation. Let $\sim$ denote the equivalence relation on $C^{0}(M, N)$, i.e. $u \sim v$ if there is a path $u^{t} \in C\left([0,1], C^{0}(M, N)\right)$ such that $u^{0}=u$ and $u^{1}=v$.

First an easy result

Theorem 0.1. Assume $p \geq \operatorname{dim} M$, then $W^{1, p}(M, N)$ is path-connected if and only if $C^{0}(M, N)$ is path-connected.

Theorem 0.1 is basically known (and relies on an idea introduced by Schoen and Uhlenbeck [SU] when $p=\operatorname{dim} M$; see also Brezis and Nirenberg [BN]). One can also deduce it from Propositions A.1, A.2 and A.3 in the Appendix.

Since, in general, $C^{0}(M, N)$ is not path-connected, this means that $W^{1, p}(M, N)$ is not path-connected when $p$ is "large". On the other hand if $p$ is "small", we expect $W^{1, p}(M, N)$ to be path-connected for all $M$ and $N$. Indeed we have

\footnotetext{
${ }^{1}$ See Remark A.1 in the Appendix if $N$ is not compact.
} 
Theorem 0.2. Let $1 \leq p<2$ (and recall that $\operatorname{dim} M \geq 2$ ). Then $W^{1, p}(M, N)$ is pathconnected.

Our proof of Theorem 0.2 is surprisingly involved and requires a number of technical tools which are presented in Sections 1-4. We call the attention of the reader especially to the "bridging" method (see Proposition 1.2 and Proposition 3.1) which is new to the best of our knowledge.

Remark 0.1. Assumption $1 \leq p<2$ in Theorem 0.2 is sharp (for general $M$ and $N$ ). For example if $\Lambda$ is any open connected set (or a connected Riemannian manifold) of dimension $\geq 1$, then $W^{1,2}\left(\mathbb{S}^{1} \times \Lambda, \mathbb{S}^{1}\right)$ is not path-connected. This may be seen using the results of B. White [W2] or Rubinstein-Sternberg [RS]. This is also a consequence of the result in [BLMN] which we recall for the convenience of the reader. Let $\Lambda$ be a connected open set (or Riemannian manifold) of dimension $\geq 1$ and let $u \in W^{1, p}\left(\mathbb{S}^{n} \times \Lambda, \mathbb{S}^{n}\right.$ ) with $p \geq n+1$ $(n \geq 1)$. Then for a.e. $\lambda \in \Lambda$ the map $u(\cdot, \lambda): \mathbb{S}^{n} \rightarrow \mathbb{S}^{n}$ belongs to $W^{1, p}$ and thus it is continuous. So $\operatorname{deg}(u(\cdot, \lambda))$ is well-defined. In this setting, the result of $[\mathrm{BLMN}]$ asserts that this degree is independent of $\lambda$ (a.e.) and that it is stable under $W^{1, n}$ convergence. Clearly this implies that $W^{1, p}\left(\mathbb{S}^{n} \times \Lambda, \mathbb{S}^{n}\right)$ is not path-connected for $p \geq n+1$.

Our next result is a generalization of Theorem 0.2.

Theorem 0.3. Let $1 \leq p<\operatorname{dim} M$, and assume that $N$ is $[p-1]$-connected, i.e.

$$
\pi_{0}(N)=\cdots=\pi_{[p-1]}(N)=0
$$

Then $W^{1, p}(M, N)$ is path-connected.

An immediate consequence of Theorem 0.3 is

Corollary 0.1. For $1 \leq p<n, W^{1, p}\left(\mathbb{S}^{n}, \mathbb{S}^{n}\right)$ is path-connected.

Remark 0.2. If $1 \leq p<2$ (i.e. the setting of Theorem 0.2) then the hypothesis on $N$ in Theorem 0.3 reads $\pi_{0}(N)=0$, i.e. $N$ is connected (which is always assumed), and thus Theorem 0.3 implies Theorem 0.2. Assumption $p<\operatorname{dim} M$ is sharp. Just take $M=N=\mathbb{S}^{n}$ and $p=n$, and recall (see e.g. $\left.[\mathrm{BN}]\right)$ that $W^{1, n}\left(\mathbb{S}^{n}, \mathbb{S}^{n}\right)$ is not path-connected since a degree is well-defined.

Corollary 0.1 may also be derived from the following general result (which is proved in Section 1.6).

Proposition 0.1. For any $1 \leq p<n$ and any $N, W^{1, p}\left(\mathbb{S}^{n}, N\right)$ is path-connected.

In the same spirit we also have

Proposition 0.2. For any $m \geq 1$, any $1 \leq p<n+1$ and any $N, W^{1, p}\left(\mathbb{S}^{n} \times B_{1}^{m}, N\right)$ is path-connected.

Here $B_{1}^{m}$ is the unit ball in $\mathbb{R}^{m}$. 
Remark 0.3. As in Remark 0.1, assumption $p<n+1$ is optimal since $W^{1, p}\left(\mathbb{S}^{n} \times B_{1}^{m}, N\right)$ is not path-connected when $p \geq n+1$ and $\pi_{n}(N) \neq 0$. This is again a consequence of a result in $[\mathrm{BLMN}]$ (Section 2, Theorem $2^{\prime}$ ).

An interesting problem which we have not settled is the following

Conjecture 1. Given $u \in W^{1, p}(M, N)$ (any $1 \leq p<\infty$, any $M$, any $N$ ), there exists a $v \in C^{\infty}(M, N)$ and a path $u^{t} \in C\left([0,1], W^{1, p}(M, N)\right)$ such that $u^{0}=u$ and $u^{1}=v$.

We have strong evidence that the above conjecture is true. First observe that if $p \geq$ $\operatorname{dim} M$, Conjecture 1 holds (this is a consequence of Proposition A.2 in the Appendix). Next, it is a consequence of Theorem 0.2 that the conjecture holds when $\operatorname{dim} M=2$. Indeed if $p<2$, any $u$ may be connected to a constant map; if $p \geq 2=\operatorname{dim} M$ we are again in the situation just mentioned above. Conjecture 1 also holds when $M=\mathbb{S}^{n}$ (any $p$ and any $N$ ); this is a consequence of Proposition 0.1 when $p<n$.

Here are two additional results in support of Conjecture 1.

Theorem 0.4. If $\operatorname{dim} M=3$ and $\partial M \neq \emptyset$ (any $N$ and any $p)$, Conjecture 1 holds.

Theorem 0.5. If $N=\mathbb{S}^{1}$ (any $M$ and any $p$ ), Conjecture 1 holds.

Theorem 0.4 is proved in Section 6 and Theorem 0.5 is proved in Section 7.

Next we analyze how the topology of $W^{1, p}(M, N)$ "deteriorates" as $p$ decreases from infinity to 1 . We denote by $[u]$ and $[u]_{p}$ the equivalence classes associated with $\sim$ and $\sim_{p}$. It is not difficult to see (Proposition A.1 in the Appendix) that if $u, v \in W^{1, p}(M, N) \cap$ $C^{0}(M, N), 1 \leq p<\infty$, with $u \sim v$, then $u \sim_{p} v$. As a consequence we have a well-defined map

$$
i_{p}:[u] \rightarrow[u]_{p}
$$

going from $C^{1}(M, N) / \sim$ to $W^{1, p}(M, N) / \sim_{p}$.

The following definition is natural:

Definition 0.1. If $i_{p}$ is bijective, we say that $W^{1, p}(M, N)$ and $C^{0}(M, N)$ have the same topology (or more precisely the same homotopy classes).

In the Appendix, we show

Proposition 0.3. For $p \geq \operatorname{dim} M, W^{1, p}(M, N)$ and $C^{0}(M, N)$ have the same topology.

Another, much more delicate, case where $W^{1, p}(M, N)$ and $C^{0}(M, N)$ have the same topology is

Theorem 0.6. For any $p \geq 2$ and any $M, W^{1, p}\left(M, \mathbb{S}^{1}\right)$ and $C^{0}\left(M, \mathbb{S}^{1}\right)$ have the same topology.

Remark 0.4. On the other hand, $W^{1, p}\left(M, \mathbb{S}^{1}\right)$ and $C^{0}\left(M, \mathbb{S}^{1}\right)$ do not have the same topology for $p<2$ if $C^{0}\left(M, \mathbb{S}^{1}\right)$ is not path-connected; this is a consequence of Theorem 0.2. 
For $q \geq p$ we also have a well-defined map

$$
i_{p, q}: W^{1, q}(M, N) / \sim_{q} \rightarrow W^{1, p}(M, N) / \sim_{p}
$$

It is then natural to introduce the following

Definition 0.2. Let $1<p<\infty$. We say that a change of topology occurs at $p$ if for every $0<\epsilon<p-1, i_{p-\epsilon, p+\epsilon}$ is not bijective. Otherwise we say that there is no change of topology at $p$. We denote by $C T(M, N)$ the set of $p^{\prime} s$ where a change of topology occurs.

Note that if $p>1$ is not in $C T$, then there exists $0<\bar{\epsilon}<p-1$ such that $i_{p_{1}, p_{2}}$ is bijective for all $p-\bar{\epsilon}<p_{1}<p_{2}<p+\bar{\epsilon}$. Consequently, $C T$ is closed. In fact we have the following property of $C T(M, N)$ which relies on Theorem 0.2 .

Proposition 0.4. $C T(M, N)$ is a compact subset of $[2, \operatorname{dim} M]$.

Remark 0.5. Assuming that Conjecture 1 holds, then $i_{p, q}$ is always surjective. As a consequence, a change of topology occurs at $p$ if for every $0<\epsilon<p-1, i_{p-\epsilon, p+\epsilon}$ is not injective, i.e., for every $0<\epsilon<p-1$, there exist $u_{\epsilon}$ and $v_{\epsilon}$ in $C^{1}$ such that $\left[u_{\epsilon}\right]_{p-\epsilon}=\left[v_{\epsilon}\right]_{p-\epsilon}$ while $\left[u_{\epsilon}\right]_{p+\epsilon} \neq\left[v_{\epsilon}\right]_{p+\epsilon}$.

Another consequence of Theorem 0.2 is

Proposition 0.5. If $C T(M, N)=\emptyset$ then $C^{0}(M, N)$ and $W^{1, p}(M, N)$ are path-connected for all $p \geq 1$.

Remark 0.6. Assuming that Conjecture 1 holds, then the following statements are equivalent:

a) $C T(M, N)=\emptyset$.

b) $C^{0}(M, N)$ is path-connected.

c) $W^{1, p}(M, N)$ is path-connected for all $p \geq 1$.

Here is another very interesting conjecture

\section{Conjecture 2.}

$$
C T(M, N) \subset\{2,3, \cdots, \operatorname{dim} M\} .
$$

A stronger form of Conjecture 2 is

Conjecture $\mathbf{2}^{\prime}$. For every integer $j \geq 1$ and any $p, q$ with $j \leq p \leq q<j+1, i_{p, q}$ is bijective.

Remark 0.7. If Conjecture 1 holds, then Conjecture $2^{\prime}$ can be stated as follows: assume $u, v \in W^{1, p}(M, N)$ (any $p$, any $M$, and any $N$ ) are homotopic in $W^{1,[p]}(M, N)$, then they are homotopic in $W^{1, p}(M, N)$.

In connection with Conjecture 2 we may also raise the following 
Open problem. Is it true that for any $n \geq 2$ and any $\Gamma \subset\{2,3, \cdots, n\}$, there exist $M$ and $N$ such that $\operatorname{dim} M=n$ and

$$
C T(M, N)=\Gamma ?
$$

We list some more properties of $C T(M, N)$ which will be discussed in Section 8:

1) For all $N$,

$$
C T\left(B_{1}^{n}, N\right)=\emptyset
$$

2) For all $N$,

$$
C T\left(\mathbb{S}^{n}, N\right)= \begin{cases}\{n\}, & \text { if } \pi_{n}(N) \neq 0 \\ \emptyset, & \text { if } \pi_{n}(N)=0\end{cases}
$$

In particular,

$$
C T\left(\mathbb{S}^{n}, \mathbb{S}^{n}\right)=\{n\}
$$

3) For all $M$,

$$
C T\left(M, \mathbb{S}^{1}\right)=\left\{\begin{array}{ll}
\{2\}, & \text { if } C^{0}\left(M, \mathbb{S}^{1}\right) \text { is not path-connected, } \\
\emptyset, & \text { if } C^{0}\left(M, \mathbb{S}^{1}\right) \text { is path-connected. }
\end{array} .\right.
$$

4) If $C T(M, N)$ is non-empty and $\pi_{0}(N)=\cdots=\pi_{k}(N)=0$ for some $k \geq 0$, then

$$
\min \{p ; p \in C T(M, N)\} \geq \min \{k+2, \operatorname{dim} M\} \text {. }
$$

5) If $\Lambda$ is compact and connected with $\operatorname{dim} \Lambda \geq 1$, then

$$
\min \left\{p ; p \in C T\left(\mathbb{S}^{n} \times \Lambda, \mathbb{S}^{n}\right)\right\}=n+1, \quad n \geq 1
$$

It would be interesting to determine $C T(M, N)$ in some concrete cases, e.g. $M$ and $N$ are products of spheres. We plan to return to this question in the future.

In this paper we have investigated the structure of the path-connected components of $W^{1, p}(M, N)$, i.e. $\pi_{0}\left(W^{1, p}(M, N)\right)$. It would be interesting to analyze $\pi_{k}\left(W^{1, p}(M, N)\right)$ for $k \geq 1$, starting from $\pi_{1}\left(W^{1, p}(M, N)\right)$. Of course it is natural to consider first the case where $1 \leq p<2$ since we already know that $W^{1, p}$ is path-connected.

Warning: People have considered several spaces of maps closely related to $W^{1, p}(M, N)$ (see e.g. White [W1] and [W2]), for example

$$
Z^{1, p}(M, N)=\text { the closure in } W^{1, p} \text { of } C^{\infty}(M, N) .
$$


This is a subset of $W^{1, p}(M, N)$ and in general a strict subset (see Bethuel [B]). One may ask the same questions as above (i.e. path-connectedness, etc.) for $Z^{1, p}(M, N)$. We warn the reader that the properties of $Z^{1, p}(M, N)$ may be quite different from the properties of $W^{1, p}(M, N)$. For example, if $1 \leq p<2$, then $W^{1, p}\left(\mathbb{S}^{1} \times \Lambda, \mathbb{S}^{1}\right)(\Lambda$ connected, $\operatorname{dim} \Lambda \geq 1)$ is path-connected by Theorem 0.2 . On the other hand $Z^{1, p}\left(\mathbb{S}^{1} \times \Lambda, \mathbb{S}^{1}\right)$ is not path-connected. Indeed, note that if $u \in C^{\infty}\left(\mathbb{S}^{1} \times \Lambda, \mathbb{S}^{1}\right)$ then

$$
\psi(u):=\oint_{\Lambda} f_{\mathbb{S}^{1}}\left(u \times u_{\theta}\right) d \theta d \lambda \in \mathbb{Z}
$$

(and $\psi(u)$ represents the degree of the map $u(\cdot, \lambda)$ for any $\lambda \in \Lambda$ ). By density $\psi(u) \in \mathbb{Z}$ for all $u \in Z^{1, p}\left(\mathbb{S}^{1} \times \Lambda, \mathbb{S}^{1}\right)$ and since $\psi$ can take any integer value it follows that $Z^{1, p}$ is not path-connected.

F. Bethuel $[\mathrm{B}]$ has been mostly concerned with the question of density of smooth maps in $W^{1, p}(M, N)$. B. White [W2] deals with the question of how much the topological properties are preserved by $W^{1, p}$ (or $Z^{1, p}$, etc.). We have tried to analyze how much of the topology "deteriorates" when passing to $W^{1, p}$, i.e., whether two smooth maps $u, v \in C^{\infty}(M, N)$ in different homotopy classes (in the usual sense) can nevertheless be connected in $W^{1, p}$ for appropriate $p^{\prime}$ s. Roughly speaking our concerns complement those of B. White as well as those in [BLMN]. However some of our techniques resemble those of B. White and F. Bethuel.

The plan of the paper is as follows.

$\S 0$. Introduction.

$\S 1$. Some useful tools. Proof of Proposition 0.1

$\S 2$. Proof of Theorem 0.2 when $\operatorname{dim} M=2$.

$\S 3$. Some more tools. Proof of Proposition 0.2

$\S 4$. Proof of Theorem 0.2 when $\operatorname{dim} M \geq 3$.

$\S 5$. Proof of Theorem 0.3 .

$\S 6$. Evidence in support of Conjecture 1: Proof of Theorem 0.4.

$\S 7$. Everything you wanted to know about $W^{1, p}\left(M, \mathbb{S}^{1}\right)$.

$\S 8$. Some properties of $C T(M, N)$.

Appendix.

Acknowledgment. We thank P. Mironescu, S. Ferry, F. Luo, and C. Weibel for useful conversations. The first author (H.B.) is partially supported by the European Grant ERB FMRX CT98 0201. He is also a member of the Institut Universitaire de France. The second author (Y.L.) is partially supported by the Grant NSF-DMS-9706887. 


\section{Section 1. Some Useful Tools}

In this section we present various techniques for connecting continuously in $W^{1, p}$ a given map to another map with desired properties. Here is a list of contents.

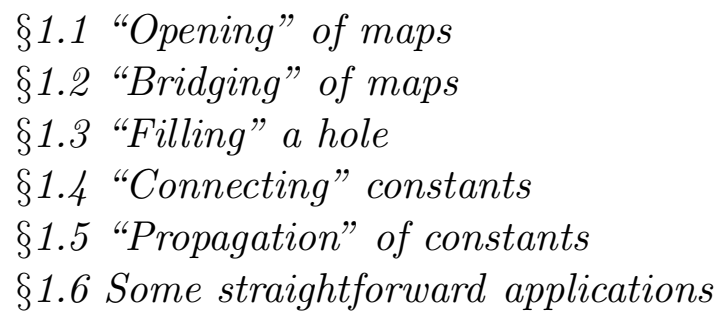

\section{$\S 1.1$ "Opening" of maps.}

Let $u$ belong to $W^{1, p}\left(\mathbb{R}^{n}, N\right)$ where $N$ is some $k$-dimensional Riemannian manifold, and $1 \leq p<\infty$. The purpose of this operation is first to construct a function $v$ which belongs to $W^{1, p}\left(\mathbb{R}^{n}, N\right)$ such that, for some point $a \in \mathbb{R}^{n}$,

1) $v(x)=u(x) \quad$ for $|x-a|>2$,

2) $v(x)=$ constant for $|x-a|<1$,

and to connect by homotopy the given $u$ to this $v$. In this case we will say that we have opened the map $u$ at the point $a$. This type of construction will be used frequently to connect a given map continuously to a constant within the space $W^{1, p}\left(\mathbb{R}^{n}, N\right)$, also when $\mathbb{R}^{n}$ is replaced by more general domains or manifolds.

We start with the construction of $v$. We will always use $B_{r}$ to denote the ball in $\mathbb{R}^{n}$ of radius $r$ and centered at the origin, unless otherwise stated.

Lemma 1.1. Let $u \in W^{1, p}\left(B_{4}\right), \quad p \geq 1, n \geq 1$. Assume

$$
\int_{B_{4}} \frac{|\nabla u(x)|^{p}}{|x|^{n-1}} d x<\infty .
$$

Then 0 is a Lebesgue-point of $u$, and in polar coordinates, with $r=|x|$ and $\sigma=\frac{x}{|x|}$,

$$
v(x):= \begin{cases}u(0), & |x| \leq 1 \\ u(2 r-2, \sigma), & 1<|x|<2 \\ u(x), & 2 \leq|x|<4\end{cases}
$$

is in $W^{1, p}\left(B_{4}\right)$.

Proof. We split the argument into 4 steps. 
Step 1. We claim that

$$
f_{B_{\varepsilon}}\left|u-\oint_{B_{\varepsilon}} u\right| \leq C \int_{B_{\varepsilon}} \frac{|\nabla u(x)|}{|x|^{n-1}}
$$

where $C$ is some constant depending only on $n$.

Proof. By Poincaré inequality,

$$
\int_{B_{1}}\left|u-\oint_{B_{1}} u\right| \leq C \int_{B_{1}}|\nabla u(x)|
$$

and therefore

$$
\int_{B_{1}}\left|u-f_{B_{1}} u\right| \leq C \int_{B_{1}} \frac{|\nabla u(x)|}{|x|^{n-1}}
$$

Estimate (1.2) follows from the above by scaling.

Step 2. Under the assumption of Lemma 1.1,

$$
\lim _{\varepsilon \rightarrow 0} \int_{\partial B_{\varepsilon}} u \quad \text { exists }
$$

and therefore

$$
\lim _{\varepsilon \rightarrow 0} \int_{B_{\varepsilon}} u \quad \text { exists. }
$$

Proof. Set

$$
w(r)=\oint_{\partial B_{r}} u
$$

Then, in polar coordinates,

$$
w^{\prime}(r)=\oint_{\mathbb{S}^{n-1}} u_{r}(r, \sigma) d \sigma
$$

and therefore

$$
\int_{0}^{1}\left|w^{\prime}(r)\right| d r \leq C \oint_{B_{1}} \frac{|\nabla u(x)|}{|x|^{n-1}} .
$$

Hence (1.3) holds, and (1.4) is an immediate consequence.

Step 3. 0 is a Lebesgue point of $u$. 
Proof. By Step 1 we have, for all $c \in \mathbb{R}$,

$$
\oint_{B_{\varepsilon}}|u-c| \leq C \int_{B_{\varepsilon}} \frac{|\nabla u(x)|}{|x|^{n-1}}+\left|c-\oint_{B_{\varepsilon}} u\right|
$$

Choosing $c=\lim _{\varepsilon \rightarrow 0} \int_{B_{\varepsilon}} u$, we find that 0 is a Lebesgue point of $u$.

Step 4. $v$ is in $W^{1, p}\left(B_{4}\right)$.

Proof. A simple calculation yields

$$
\begin{aligned}
\int_{B_{2} \backslash B_{1}}|v| & =\int_{\mathbb{S}^{n-1}} \int_{1}^{2}|v(r, \sigma)| r^{n-1} d r d \sigma \\
& \leq C \int_{\mathbb{S}^{n-1}} \int_{0}^{2} \frac{|u(s, \sigma)|}{s^{n-1}} s^{n-1} d s d \sigma=C \int_{B_{2}} \frac{|u(x)|}{|x|^{n-1}}
\end{aligned}
$$

We also have

$$
\begin{aligned}
\int_{B_{2}} \frac{|u(x)|}{|x|^{n-1}} & =\int_{0}^{2} \frac{1}{r^{n-1}}\left[\int_{\partial B_{r}}|u|\right] d r \leq \frac{1}{2^{n-1}} \int_{B_{2}}|u| \\
& +(n-1) \int_{0}^{2}\left[\frac{1}{r^{n}} \int_{B_{r}}|u|\right] d r .
\end{aligned}
$$

Since 0 is a Lebesgue point, $\lim _{r \rightarrow \infty} \frac{1}{r^{n}} \int_{B_{r}}|u-u(0)|=0$, and therefore the second integral on the right-hand side is finite and thus

$$
\int_{B_{2} \backslash B_{1}}|v|<\infty
$$

Similarly,

$$
\int_{B_{2} \backslash B_{1}}|\nabla v|^{p} \leq C \int_{\mathbb{S}^{n-1}} \int_{0}^{2} \frac{|\nabla u(s, \sigma)|^{p} s^{n-1}}{s^{n-1}} d s d \sigma \leq C \int_{B_{2}} \frac{|\nabla u(x)|^{p}}{|x|^{n-1}}<\infty,
$$

by (1.1). Combining this with (1.6) we obtain that $v \in W^{1, p}\left(B_{2} \backslash B_{1}\right)$.

To show that $v \in W^{1, p}\left(B_{4}\right)$ we only need to verify on $\partial B_{1}$, in the sense of trace, that $v-u(0)=0$. For $1<r<2$, with $s=2 r-2$, we have

$$
\int_{\partial B_{r}}|v-u(0)|=\left(\frac{r}{s}\right)^{n-1} \int_{\partial B_{s}}|u-u(0)| \leq\left(\frac{2}{s}\right)^{n-1} \int_{\partial B_{s}}|u-u(0)|
$$


and, since $x=0$ is a Lebesgue point of $u$,

$$
\frac{1}{s^{n}} \int_{0}^{s}\left\{\int_{\partial B_{\mu}}|u-u(0)|\right\} d \mu=\frac{1}{s^{n}} \int_{B_{s}}|u-u(0)| \rightarrow 0, \text { as } s \rightarrow 0 .
$$

So, along a subsequence $s_{i} \rightarrow 0$,

$$
\lim _{i \rightarrow 0} \int_{\partial B_{r_{i}}}|v-u(0)|=\lim _{i \rightarrow \infty} \frac{1}{s_{i}^{n-1}} \int_{\partial B_{s_{i}}}|u-u(0)|=0,
$$

where $r_{i}=\frac{1}{2}\left(s_{i}+2\right) \rightarrow 1^{+}$. Lemma 1.1 is established.

Remark 1.1. If condition (1.1) is replaced by

$$
\int_{B_{4}} \frac{|\nabla u(x)|^{p}}{|x-a|^{n-1}} d x<\infty
$$

for some $|a|<1$, then the conclusion of Lemma 1.1 holds with the origin shifted to $a$, with $v$ defined in $B_{3}$ instead of $B_{4}$. Note that by Fubini's theorem, if $u \in W^{1, p}\left(B_{4}\right)$, then almost all points $a$ in $B_{1}$ satisfy (1.7). Such a point will be called a "good" point.

Our next result provides a homotopy connecting a given map $u$ to the map $v$ constructed in the previous lemma.

Proposition 1.1. Under the hypotheses of Lemma 1.1, set, for $0<t \leq 1$,

$$
u^{t}(x):= \begin{cases}u(0), & |x| \leq t, \\ u(2 r-2 t, \sigma), & t<|x| \leq 2 t, \\ u(x), & 2 t \leq|x| \leq 4,\end{cases}
$$

and $u^{0}=u$. Then

$$
u^{t} \in C\left([0,1], W^{1, p}\left(B_{4}\right)\right) .
$$

Proof. By Lemma 1.1, $u^{t}$ is well-defined and, by standard arguments, is continuous for $t \in(0,1]$. We only need to show that $u^{t} \rightarrow u$ in $W^{1, p}\left(B_{4}\right)$ as $t \rightarrow 0^{+}$. In view of the expression of $u^{t}$, this amounts to showing

$$
\lim _{t \rightarrow 0^{+}}\left\|u^{t}\right\|_{W^{1, p}\{t \leq|x| \leq 2 t\}}=0 .
$$

An easy calculation yields

$$
\int_{t \leq|x| \leq 2 t}\left|u^{t}\right| \leq C t^{n-1} \int_{|y| \leq 2 t} \frac{|u(y)|}{|y|^{n-1}},
$$

and

$$
\int_{t \leq|x| \leq 2 t}\left|\nabla u^{t}\right|^{p} \leq C t^{n-1} \int_{|y| \leq 2 t} \frac{|\nabla u(y)|^{p}}{|y|^{n-1}} .
$$

Assertion (1.8) follows from the above, (1.1) and (1.5). Proposition 1.1 is established. 


\section{$\S 1.2$ "Bridging" of maps.}

To simplify the presentation we explain first the construction in the easy 2-dimensional case.

Consider the square

$$
\Omega=\left\{x=\left(x_{1}, x_{2}\right) ;\left|x_{1}\right|<20,\left|x_{2}\right|<20\right\}
$$

and let

$$
u \in W^{1, p}(\Omega, N)
$$

where $N$ is any smooth (connected) Riemannian manifold with or without boundary of dimension $\geq 1$.

We assume that $u$ is constant, say $Y_{0}$, in the region $Q^{+} \cup Q^{-}$where

$$
Q^{+}=\left\{x=\left(x_{1}, x_{2}\right) ;\left|x_{1}\right|<20,1<x_{2}<20\right\}
$$

and

$$
Q^{-}=\left\{x=\left(x_{1}, x_{2}\right) ;\left|x_{1}\right|<20,-20<x_{2}<-1\right\} .
$$

Our purpose is to construct a map $v$ in $W^{1, p}(\Omega, N)$ such that

$$
\begin{cases}v(x)=u(x) & \text { outside }(-5,5) \times(-1,1) \\ v(x)=Y_{0} & \text { for }\left|x_{1}\right|<1 \text { and }\left|x_{2}\right|<20\end{cases}
$$

and a homotopy connecting the given $u$ to this $v$ continuously in $W^{1, p}(\Omega, N)$ and which preserves $u$ outside $(-5,5) \times(-1,1)$. We call this a "bridge" because the regions $Q^{+}$and $Q^{-}$where $u=Y_{0}$ which were originally disconnected have now become connected through the "bridge" $(-1,1) \times(-20,20)$.

Proposition 1.2. Take $\Omega$ and $u$ as above with

$$
1 \leq p<2
$$

Then there exists

$$
u^{t} \in C\left([0,1], W^{1, p}(\Omega, N)\right)
$$

such that

$$
\begin{aligned}
u^{0} & =u, \\
u^{t}(x) & =u(x), \quad \forall t \in[0,1], \quad \forall x \quad \text { outside }(-5,5) \times(-1,1), \\
u^{1}(x) & =Y_{0}, \quad \forall x \in(-1,1) \times(-20,20) .
\end{aligned}
$$


Proof. As in Remark 1.1 we may assume without loss of generality (after shifting the origin in the $x_{1}$-direction) that

$$
\int_{\Omega} \frac{|\nabla u(x)|^{p}}{\left|x_{1}\right|^{p-1}} d x_{1} d x_{2}<\infty
$$

Here we use the fact that $p<2$.

Define for $0 \leq t \leq 1, x=\left(x_{1}, x_{2}\right) \in \Omega$,

$$
v^{t}\left(x_{1}, x_{2}\right)=\tilde{u}\left(x_{1}, \frac{x_{2}}{1-t \rho\left(x_{1}\right)}\right)
$$

where $\tilde{u}$, defined in $(-20,20) \times \mathbb{R}$, is the extension of $u$ taking the value $Y_{0}$ in $\left\{\left(x_{1}, x_{2}\right) ;\left|x_{1}\right|<\right.$ $\left.20,\left|x_{2}\right| \geq 20\right\}$ and $\rho\left(x_{1}\right)=\left(1-\left|x_{1}\right|\right)^{+}$.

Clearly $v^{t} \in C\left([0,1), W^{1, p}(\Omega, N)\right)$ and satisfies (1.10), (1.11) (with $u^{t}$ replaced by $v^{t}$ ). Next, we check that $v^{t}$ is continuous at $t=1$. Fix any $\delta>0$; it is clear that $v^{t} \rightarrow v^{1}$ in $W^{1, p}$ outside $\Omega_{\delta}=\left\{\left(x_{1}, x_{2}\right) \in \Omega ;\left|x_{1}\right|<\delta\right\}$. Hence it suffices to show that

$$
\sup _{0<t \leq 1}\left\|v^{t}\right\|_{W^{1, p}\left(\Omega_{\delta}\right)} \rightarrow 0 \quad \text { as } \delta \rightarrow 0
$$

For this purpose we make a change of variables

$$
\left\{\begin{array}{l}
\xi_{1}=x_{1}, \\
\xi_{2}=\frac{x_{2}}{1-t \rho\left(x_{1}\right)},
\end{array}\right.
$$

so that the Jacobian

$$
\frac{\partial\left(\xi_{1}, \xi_{2}\right)}{\partial\left(x_{1}, x_{2}\right)}=\frac{1}{1-t \rho\left(x_{1}\right)} \geq 1
$$

Therefore, as $\delta \rightarrow 0$,

$$
\begin{aligned}
\int_{\Omega_{\delta}}\left|v^{t}(x)\right|^{p} d x & =\int_{\left|\xi_{1}\right|<\delta}|u(\xi)|^{p} \frac{\partial\left(x_{1}, x_{2}\right)}{\partial\left(\xi_{1}, \xi_{2}\right)} d \xi \\
& \leq \int_{\left|\xi_{1}\right|<\delta}|u(\xi)|^{p} d \xi \rightarrow 0 \text { uniformly in } t
\end{aligned}
$$

Next, it is easy to verify that

$$
\left|\nabla v^{t}(x)\right| \leq \frac{C|\nabla u(\xi)|}{1-t \rho\left(\xi_{1}\right)},
$$


since $\nabla v^{t}(x)=0$ if $\left|x_{2}\right|>1-t \rho\left(x_{1}\right)$.

It follows that, as $\delta \rightarrow 0$,

$$
\begin{aligned}
\int_{\Omega_{\delta}}\left|\nabla v^{t}(x)\right|^{p} d x & \leq C \int_{\left|\xi_{1}\right|<\delta} \frac{|\nabla u(\xi)|^{p}}{\left(1-t \rho\left(\xi_{1}\right)\right)^{p}}\left(1-t \rho\left(\xi_{1}\right)\right) d \xi \\
& \leq C \int_{\left|\xi_{1}\right|<\delta} \frac{|\nabla u(\xi)|^{p}}{\left|\xi_{1}\right|^{p-1}} d \xi \rightarrow 0 .
\end{aligned}
$$

Here we have used (1.13).

To summarize, we have connected $u$ to $v^{1}$ through a homotopy satisfying (1.11). Moreover $v^{1}$ satisfies also

$$
v^{1}(x)=Y_{0} \quad \forall \quad\left|x_{2}\right|>\left|x_{1}\right| .
$$

The final step is to connect this $v^{1}$, through a homotopy $w^{t}$ satisfying (1.11), to some $u^{1}$ satisfying (1.12). This can be achieved by choosing, for example,

$$
w^{t}\left(x_{1}, x_{2}\right)= \begin{cases}Y_{0} & \left|x_{1}\right|<t \rho\left(2 x_{1}\right) \\ v^{1}\left(x_{1}-t \rho\left(2 x_{1}\right), x_{2}\right) & \left|x_{1}\right| \geq t \rho\left(2 x_{1}\right) .\end{cases}
$$

Remark 1.2. The conclusion of Proposition 1.2 fails when $p \geq 2$ and $N=\mathbb{S}^{1}$. We argue by contradiction. Suppose that the conclusion holds. We may think of the maps $u$ satisfying the conditions of the proposition as defined on the annulus $A=\{(r, \theta) \mid 1<r<2,0<\theta \leq$ $2 \pi\}$, which are equal to $Y_{0}$ outside the sector $0<\theta<\theta_{0}<2 \pi$. On the other hand, the $u^{1}$ in the conclusion of the proposition is equal to $Y_{0}$ in a small annulus $\frac{5}{4}<r<\frac{3}{2}$. To reach a contradiction, we invoke the result in [BLMN] which allows to define a degree for every map $u \in W^{1, p}\left(A, \mathbb{S}^{1}\right), p \geq 2$. The degree is invariant under homotopy within $W^{1, p}\left(A, \mathbb{S}^{1}\right)$. We may start with some $u \in W^{1, p}\left(A, \mathbb{S}^{1}\right), p \geq 2$, having nonzero degree, ending up with $u^{1}$ having zero degree.

\section{$\S 1.3$ "Filling" a hole.}

Let $B$ be the unit ball in $\mathbb{R}^{n}, u \in W^{1, p}(B, N), 1 \leq p<n$, be such that

$$
u=Y_{0} \quad \text { on } \partial B
$$

for some $Y_{0} \in N$. Then $u$ can be connected in $W^{1, p}(B, N)$ to the constant map $Y_{0}$ through a homotopy which preserves the boundary condition (1.15). More precisely, we have

Proposition 1.3. Take $B$ and $u$ as above, and

$$
1 \leq p<n
$$


Then there exists

$$
u^{t} \in C\left([0,1], W^{1, p}(B, N)\right)
$$

such that

$$
u^{0}=u, \quad u^{1} \equiv Y_{0}
$$

and

$$
u^{t}(x)=Y_{0} \quad \forall 0 \leq t \leq 1 \text { and } x \in \partial B
$$

Proof. Let $\tilde{u}$ be the extension of $u$ to $\mathbb{R}^{n}$ by taking $Y_{0}$ outside $B$, and let

$$
u^{t}(x)=\tilde{u}\left(\frac{x}{1-t}\right)
$$

To complete the proof we only need to verify that $u^{t} \rightarrow Y_{0}$ in $W^{1, p}$ as $t \rightarrow 1$. Since $u^{t}$ and $Y_{0}$ have the same boundary condition, it suffices to show that $\left\|\nabla\left(u^{t}-Y_{0}\right)\right\|_{L^{p}(B)}=$ $\left\|\nabla u^{t}\right\|_{L^{p}(B)} \rightarrow 0$.

This can be easily seen from

$$
\int_{B}\left|\nabla u^{t}\right|^{p}=(1-t)^{n-p} \int_{B}|\nabla u|^{p}
$$

Remark 1.3. The conclusion of Proposition 1.3 no longer holds if we take $p \geq n$ and $\pi_{n}(N) \neq \emptyset$. Indeed, fix some continuous $\varphi$ from $\mathbb{S}^{n}$ to $N$ which is not homotopic to a constant. We can always assume that $\varphi$ is smooth. Fix any point $x_{0} \in \mathbb{S}^{n}$ and set $Y_{0}=\varphi\left(x_{0}\right)$; we may assume, after a smooth homotopy, that $\varphi(x)=Y_{0}$ for $x$ near $x_{0}$, say $x \in B_{r}\left(x_{0}\right)$ for some $r>0$. Since $\mathbb{S}^{n} \backslash B_{r}\left(x_{0}\right)$ is diffeomorphic to the unit ball $B$ of $\mathbb{R}^{n}$, the conclusion of Proposition 1.3 holds there and allows to connect $\varphi$ to $Y_{0}$ through a homotopy in $W^{1, p}\left(\mathbb{S}^{n} \backslash B_{r}\left(x_{0}\right)\right)$ which is equal to $Y_{0}$ on $\partial\left(\mathbb{S}^{n} \backslash B_{r}\left(x_{0}\right)\right)$. This yields a homotopy of $\varphi$ to a constant in $W^{1, p}\left(\mathbb{S}^{n}, N\right)$. For $p>n$, this, combined with the Sobolev embedding, contradicts the assumption that $\varphi$ is not trivial. When $p=n$, we use the embedding of $W^{1, n}$ into VMO and complete the argument as in [BN].

\section{$\S 1.4$ "Connecting" Constants.}

The purpose of the simple construction below is to homotopy a given map $u$ which is a constant $Y_{0}$ on some compact set $K$ to a map $v$ which equals another given constant $Y_{1}$ on $K$, while preserving through the homotopy the values of $u$ outside a given neighborhood of $K$. 
Proposition 1.4. Let $K$ be any compact subset of $M, \varepsilon>0, Y_{0} \in N, 1 \leq p<\infty, u \in$ $W^{1, p}(M, N)$, and

$$
u(x)=Y_{0}, \quad \text { if } \operatorname{dist}(x, K) \leq \varepsilon .
$$

Then, given any $Y_{1} \in N$, there exists

$$
u^{t} \in C\left([0,1], W^{1, p}(M, N)\right)
$$

such that

$$
\begin{gathered}
u^{0}=u, \\
u^{t}(x)=u(x), \quad \forall t \in[0,1], \quad \text { if } \operatorname{dist}(x, K)>\varepsilon / 2, \\
u^{1}(x)=Y_{1}, \quad \text { if } \operatorname{dist}(x, K)<\varepsilon / 4 .
\end{gathered}
$$

Proof. Let $f \in C^{\infty}([0,1], N)$ such that $f(0)=Y_{0}, f(1)=Y_{1}$.

Take $\rho \in C^{\infty}(M)$ such that $0 \leq \rho \leq 1$

$$
\rho(x)= \begin{cases}1 & \text { if } \operatorname{dist}(x, K) \leq \varepsilon / 4 \\ 0 & \text { if } \operatorname{dist}(x, K) \geq \varepsilon / 2\end{cases}
$$

Set

$$
u^{t}(x)= \begin{cases}u(x) & \text { if } \operatorname{dist}(x, K) \geq \varepsilon, \\ f(t \rho(x)) & \text { if } \operatorname{dist}(x, K)<\varepsilon .\end{cases}
$$

This is a desired homotopy.

\section{$\S 1.5$ "Propagation" of constants.}

The purpose of this construction is to homotopy a given $u$, which is constant in some initial region, to a map $v$ which is the same constant in a larger region, while preserving $u$ "away" from the larger region. Here, the initial region can be smoothly deformed to the larger one and thus we make no restriction on $p$. This is in contrast with the "bridging" technique above, which involves a change in topology and requires a restriction on $p$ (see e.g. Remark 1.2). To explain the construction we start with the case where the initial region is a small ball.

Proposition 1.5. Let $u \in W^{1, p}\left(B_{1}, N\right)$, where $B_{1}$ is the unit ball centered at the origin in some Euclidean space. Suppose, for some $0<\bar{\epsilon}<1$ and $Y_{0} \in N$,

$$
u(x)=Y_{0}, \quad \forall|x|<\bar{\epsilon}
$$


Then, for all $0<\epsilon<1-\bar{\epsilon}$, there exists $u^{t} \in C\left([0,1], W^{1, p}\left(B_{1}, N\right)\right)$ such that

$$
\begin{array}{cl}
u^{0}=u, & \\
u^{t}(x)=Y_{0}, & \forall 0 \leq t \leq 1,|x|<\bar{\epsilon}, \\
u^{t}(x)=u(x), & \forall 0 \leq t \leq 1,1-\frac{\epsilon}{2}<|x|<1, \\
u^{1}(x)=Y_{0}, & \forall|x|<1-\epsilon .
\end{array}
$$

Proof. Let

$$
\varphi_{t}: B_{1} \rightarrow B_{1}
$$

be a diffeomorphism which is smooth in $(t, x) \in[0,1] \times B_{1}$ having the following properties:

$$
\begin{aligned}
\varphi_{0}=i d, & \\
\varphi_{t}(x)=x, & \forall 0 \leq t \leq 1,1-\frac{\epsilon}{2}<|x|<1, \\
\left|\varphi_{t}(x)\right| \leq|x|, & \forall|x| \leq \bar{\epsilon} \\
\left|\varphi_{1}(x)\right| \leq \bar{\epsilon}, & \forall|x| \leq 1-\epsilon .
\end{aligned}
$$

Then $u^{t}:=u \circ \varphi_{t}$ is a desired homotopy.

This proposition is often used as follows. For $S \subset K \subset M, u \in W^{1, p}(M, N), u(x)=Y_{0}$, near $S$, we would like to connect $u$ to some $v$ which is $Y_{0}$ in a $\delta$-neighborhood of $K$ while along the homotopy the values in some neighborhood of $S$ are preserved as $Y_{0}$ and the values outside the $\delta^{\prime}$-neighborhood are preserved $\left(\delta^{\prime}>\delta\right)$. Suppose that we are able to construct a diffeomorphism

$$
\psi: B_{1} \rightarrow \delta^{\prime}-\text { neighborhood of } K
$$

$B_{1} \subset \mathbb{R}^{n}, \operatorname{dim} M=n$, such that

$$
\begin{gathered}
\delta-\text { neighborhood of } K \subset \psi\left(B_{\frac{8}{9}}\right), \\
u(x)=Y_{0}, \quad \forall x \in \psi\left(B_{\frac{1}{9}}\right),
\end{gathered}
$$

and

$$
\psi\left(B_{\frac{1}{9}}\right) \text { contains some neighborhood of } S \text {. }
$$

Then we can apply the proposition to $u \circ \psi$ with $\epsilon=\bar{\epsilon}=\frac{1}{9}$.

In our later applications, the construction of $\psi$ is always obvious and we will not really construct $\psi$ explicitly but only refer to this technique as "propagation" of constants. 


\section{§1.6 Some straightforward applications.}

We now present some immediate applications of the above techniques.

Proposition 1.6. $W^{1, p}\left(B_{1}^{n}, N\right)$ is path-connected for any $n$, any $p$, and any $N$.

Proof. Let $u \in W^{1, p}\left(B_{1}^{n}, N\right)$; we first "open" the map $u$ at a "good" point near the origin (Proposition 1.1 and Remark 1.1) to connect $u$ to some $v \in W^{1, p}\left(B_{1}^{n}, N\right)$ satisfying, for some $0<r<1$ and $Y_{0} \in N$,

$$
v(x)=Y_{0} \quad \forall|x|<r .
$$

Then the homotopy $v^{t}(x)=v(t x)(r \leq t \leq 1)$ connects $v$ to $Y_{0}$. Finally, by Proposition 1.4, any two constant maps can be connected to each other.

Proposition 0.1. For any $1 \leq p<n$ and any $N, W^{1, p}\left(\mathbb{S}^{n}, N\right)$ is path-connected.

Proof of Proposition 0.1. Let $u \in W^{1, p}\left(\mathbb{S}^{n}, N\right)$. By "opening" $u$ at a "good" point, we connect $u$ to some $v \in W^{1, p}\left(\mathbb{S}^{n}, N\right)$ satisfying $v=Y_{0}$ in a geodesic ball $B_{r}$. Since $\mathbb{S}^{n} \backslash B_{r}$ is topologically a ball, we can apply Proposition 1.3 to connect $v$ to the constant map $Y_{0}$. Here we use $p<n$.

\section{Section 2. Proof of Theorem 0.2 When $\operatorname{dim} M=2$}

We discuss only the case where $\partial M=\emptyset$; for the case where $\partial M \neq \emptyset$, see Remark 2.1 at the end of this section. Consider a triangulation $\left\{T_{1}, \cdots, T_{l}\right\}$ of $M$. Let $\left\{v_{1}, \cdots, v_{k}\right\}$ be the collection of all vertices in the triangulation, and let $\left\{e_{1}, \cdots, e_{m}\right\}$ be the collection of all edges.

Our purpose is to show that any $u \in W^{1, p}(M, N)$ is homotopic to a constant. In order to connect $u$ to a constant, $Y_{0}$, we proceed in three steps. First, we connect $u$ to some $u_{0}$ which equals $Y_{0}$ near all the vertices. Then, we connect $u_{0}$ to some $u_{1}$ which equals $Y_{0}$ near all the edges. Finally, we connect $u_{1}$ to $Y_{0}$.

Step 1: Connect $u$ to $u_{0}$ which equals $Y_{0}$ near all the vertices.

This is easily done by "opening" of maps (Proposition 1.1) and "connecting" constants (Proposition 1.4).

To open the map we may always choose "good" points (in the sense of (1.7)) near the vertices and open from there.

Step 2: Connect $u_{0}$ to $u_{1}$ which equals $Y_{0}$ near all the edges.

We proceed by induction on the number of edges. First, for a single $e_{1}$, recall that $u_{0}$ equals $Y_{0}$ near $\partial e_{1}$, the two end-points of $e_{1}$. By "propagation" (Proposition 1.5) and 
"bridging" (Proposition 1.2), we connect $u_{0}$ to $u_{0,1}$ which equals $Y_{0}$ near $e_{1} \cup\{$ all vertices $\}$. To proceed with the induction, we may assume that we have connected $u_{0}$ to a map $u_{0, k}$ which equals $Y_{0}$ in an $\varepsilon$-neighborhood of $e_{1} \cup \cdots \cup e_{k} \cup\{$ all vertices $\}$. We now wish to add $e_{k+1}$ to the collection. There are three possibilities:

Case 1. $e_{k+1} \cap\left\{e_{1} \cup \cdots \cup e_{k}\right\}=\emptyset$,

Case 2. $e_{k+1} \cap\left\{e_{1} \cup \cdots \cup e_{k}\right\}=1$-vertex,

Case 3. $e_{k+1} \cap\left\{e_{1} \cup \cdots \cup e_{k}\right\}=2$-vertices.

In all cases, we can find $0<\delta \ll \varepsilon$ such that

$$
Z \cap\left\{\left\{\delta \text {-neighborhood of } e_{k+1}\right\} \backslash\left\{\frac{\varepsilon}{2} \text {-neighborhood of } \partial e_{k+1}\right\}\right\}=\emptyset,
$$

where $Z=e_{1} \cup \cdots \cup e_{k} \cup\{$ all vertices $\}$.

By "propagation" (Proposition 1.5) and "bridging" (Proposition 1.2) we end up with a map $u_{0, k+1}$ which equals $Y_{0}$ near $Z \cup e_{k+1}$. We may do so keeping $u_{0, k+1}=u_{0, k}$ outside

$$
\left\{\delta \text {-neighborhood of } e_{k+1}\right\} \backslash\left\{\frac{\varepsilon}{2} \text {-neighborhood of } \partial e_{k+1}\right\} \text {. }
$$

This completes the induction and Step 2 is finished.

Step 3: Connect $u_{1}$ to $Y_{0}$.

Recall that $u_{1}$ equals $Y_{0}$ near $\partial T_{i}$ for all $1 \leq i \leq \ell$.

Applying Proposition 1.3 ("Filling" a hole) successively on $T_{1}, \cdots, T_{l}$ yields the desired conclusion.

Remark 2.1. By a standard procedure (e.g. reflection across the boundary) we construct a smooth neighborhood $M^{\prime}$ of $M$ and an extension of $u$ to $M^{\prime}$, still denoted by $u \in$ $W^{1, p}\left(M^{\prime}, N\right)$. We then proceed as above.

\section{SECtion 3. Some more tools}

Here we return to the "bridging", "opening" and "filling" techniques described in $§ 1.1$ 1.3 , and present some refinements.

We work in $\mathbb{R}^{n}, n \geq 2$ and we distinguish some special variables. For $0 \leq \ell \leq n-2$, we write

$$
x=\left(x^{\prime}, x^{\prime \prime}\right),
$$

where $x^{\prime}=\left(x_{1}, \ldots, x_{n-\ell-1}\right), x^{\prime \prime}=\left(x_{n-\ell}, \ldots, x_{n}\right)$.

Let

$$
\Omega=\left\{\left(x^{\prime}, x^{\prime \prime}\right) ;\left|x^{\prime}\right|<20,\left|x^{\prime \prime}\right|<20\right\}
$$


Proposition 3.1. Assume $u \in W^{1, p}(\Omega, N)$,

$$
1 \leq p<\ell+2,
$$

and

$$
u(x)=Y_{0} \quad \forall x, 1<\left|x^{\prime \prime}\right|<20,\left|x^{\prime}\right|<20,
$$

for some $Y_{0} \in N$.

Then there exists $u^{t} \in C\left([0,1], W^{1, p}(\Omega, N)\right)$ such that

$$
\begin{aligned}
u^{0} & =u \\
u^{t}(x) & =u(x), \quad \forall 0 \leq t \leq 1, x \text { outside }\left\{x ;\left|x^{\prime \prime}\right|<1,\left|x^{\prime}\right|<1\right\}, \\
u^{1}(x) & =Y_{0}, \quad \forall x,\left|x^{\prime \prime}\right|<20,\left|x^{\prime}\right|<1 / 8,
\end{aligned}
$$

Remark 3.1. The case $n=2$ and $\ell=0=n-2$ corresponds to Proposition 1.2 with $x^{\prime}=x_{1}$, and $x^{\prime \prime}=x_{2}$. Assumption (3.1) is consistent with the assumption $p<2$ there.

Proof of Proposition 3.1. If $\ell=n-2$, then $x^{\prime}=x_{1}$; if $0 \leq \ell<n-2$, we write $x^{\prime}=$ $\left(x_{1}, \tilde{x}\right), \tilde{x}=\left(x_{2}, \cdots, x_{n-\ell-1}\right)$.

As in Remark 1.1, we may assume (by an appropriate selection)

$$
\int_{\Omega} \frac{|\nabla u(x)|^{p}}{\left|x_{1}\right|^{p-\ell-1}}<\infty .
$$

It is here that we use (3.1).

For $0 \leq t \leq 1, x=\left(x_{1}, \tilde{x}, x^{\prime \prime}\right) \in \Omega$, define

$$
v^{t}\left(x_{1}, \tilde{x}, x^{\prime \prime}\right)=\tilde{u}\left(x_{1}, \tilde{x}, \frac{x^{\prime \prime}}{1-t \rho\left(x_{1}\right) \eta(\tilde{x})}\right),
$$

where $\rho\left(x_{1}\right)=\left(1-\left|x_{1}\right|\right)^{+}$and

$$
\eta(\tilde{x})= \begin{cases}1 & |\tilde{x}| \leq 1 \\ 2-|\tilde{x}| & 1<|\tilde{x}|<2 \\ 0 & |\tilde{x}| \geq 2\end{cases}
$$

Here $\tilde{u}$, defined in

$$
\left\{\left(x^{\prime}, x^{\prime \prime}\right) ; \quad\left|x^{\prime}\right|<20, \quad x^{\prime \prime} \in \mathbb{R}^{\ell+1}\right\},
$$


is the extension of $u$ taking the value $Y_{0}$ in $\left\{\left(x^{\prime}, x^{\prime \prime}\right) ;\left|x^{\prime}\right|<20,\left|x^{\prime \prime}\right| \geq 20\right\}$. Clearly $v^{t} \in$ $C\left([0,1), W^{1, p}(\Omega, N)\right)$ and satisfies (3.2) and (3.3). Next we check that $v^{t}$ is continuous at $t=1$. Fix any $\delta>0$; it is clear that, as $t \rightarrow 1, v^{t} \rightarrow v^{1}$ in $W^{1, p}$ outside $\Omega_{\delta}=\left\{\left(x_{1}, \tilde{x}, x^{\prime \prime}\right) \in\right.$ $\left.\Omega ;\left|x_{1}\right|<\delta\right\}$.

Hence it suffices to show that

$$
\sup _{0<t \leq 1}\left\|v^{t}\right\|_{W^{1, p}\left(\Omega_{\delta}\right)} \rightarrow 0 \quad \text { as } \delta \rightarrow 0
$$

For this purpose we make a change of variables

$$
\left\{\begin{array}{l}
\xi_{1}=x_{1}, \\
\tilde{\xi}=\tilde{x} \\
\xi^{\prime \prime}=\frac{x^{\prime \prime}}{1-t \rho\left(x_{1}\right) \eta(\tilde{x})},
\end{array}\right.
$$

so that the Jacobian

$$
\frac{\partial\left(\xi_{1}, \tilde{\xi}, \xi^{\prime \prime}\right)}{\partial\left(x_{1}, \tilde{x}, x^{\prime \prime}\right)}=\frac{1}{\left[1-t \rho\left(x_{1}\right) \eta(\tilde{x})\right]^{\ell+1}} \geq 1
$$

Therefore, as $\delta \rightarrow 0$,

$$
\int_{\Omega_{\delta}}\left|v^{t}(x)\right|^{p} d x \rightarrow 0 \quad \text { uniformly in } t
$$

Next,

$$
\left|\nabla v^{t}(x)\right| \leq \frac{C|\nabla u(\xi)|}{\left[1-t \rho\left(\xi_{1}\right) \eta(\tilde{\xi})\right]}
$$

It follows, as $\delta \rightarrow 0$, that

$$
\begin{aligned}
\int_{\Omega_{\delta}}\left|\nabla v^{t}(x)\right|^{p} d x & \leq C \int_{\left|\xi_{1}\right|<\delta} \frac{|\nabla u(\xi)|^{p}\left[1-t \rho\left(\xi_{1}\right) \eta(\tilde{\xi})\right]^{\ell+1}}{\left[1-t \rho\left(\xi_{1}\right) \eta(\tilde{\xi})\right]^{p}} d \xi \\
& \leq C \int_{\left|\xi_{1}\right|<\delta} \frac{|\nabla u(\xi)|^{p} d \xi}{\left|1-\rho\left(\xi_{1}\right)\right|^{p-\ell-1}} \rightarrow 0 .
\end{aligned}
$$

Here we have used (3.5).

So far we have connected the original $u$ to $v^{1}$ through a homotopy satisfying (3.2), (3.3) and $v^{1}$ has the property that

$$
v^{1}\left(0, \tilde{x}, x^{\prime \prime}\right)=Y_{0} \quad \forall|\tilde{x}|<1,\left|x^{\prime \prime}\right|<20 .
$$

The final step is to connect this $v^{1}$, through a homotopy $w^{t}$ satisfying (3.3), to some $u^{1}$ satisfying (3.4). This can be achieved by choosing for example

$$
w^{t}\left(x_{1}, \tilde{x}, x^{\prime \prime}\right)= \begin{cases}Y_{0}, & \left|x_{1}\right|<t \rho\left(2 x_{1}\right) \eta(2 \tilde{x}) \\ v^{1}\left(x_{1}-t \rho\left(2 x_{1}\right) \eta(2 \tilde{x}), \tilde{x}, x^{\prime \prime}\right), & \left|x_{1}\right| \geq t \rho\left(2 x_{1}\right) \eta(2 \tilde{x}) .\end{cases}
$$


Remark 3.2. The conclusion of Proposition 3.1 no longer holds if we take $p \geq \ell+2$ and $\pi_{\ell+1}(N) \neq \emptyset$ (this can be seen as in Remark 1.2).

We now present a refinement of the "opening" technique in $\S 1.1$ which will be used in the proof of Theorem 0.3. Here the map $u$ also depends on "dummy" parameters $a \in A$; but the "opening" is done with respect to the $x$ variables.

Proposition 3.2. Let $N$ and $A$ be smooth Riemannian manifolds with or without boundary, and let $u \in W^{1, p}\left(B_{4} \times A, N\right)$ where $p \geq 1$ and $B_{4}$ is the ball in $\mathbb{R}^{n}$ of radius 4 and centered at the origin. Then there exists a continuous path $u^{t} \in C\left([0,1], W^{1, p}\left(B_{4} \times A, N\right)\right)$ such that $u^{0}=u, u^{t}(x, a)=u(x, a)$ for all $t \in[0,1], a \in A$, and $x \in B_{4} \backslash B_{2 / 3}$ and, for some $Y \in W^{1, p}(A, N), u^{1}(x, a) \equiv Y(a)$ for $a \in A$ and $x \in B_{1 / 3}$.

Remark 3.3. It is easy to see from the proof that the map $Y(a)$ can be taken as some $u(\bar{x}, a)$ with $|\bar{x}|$ as small as we wish.

The proof relies on several lemmas; the first one is an extension of Lemma 1.1.

Lemma 3.1. For $u \in W^{1, p}\left(B_{4} \times A\right), p \geq 1$. Assume

$$
\int_{B_{4} \times A} \frac{|\nabla u(x, a)|^{p}}{|x|^{n-1}} d x d a<\infty
$$

where $\nabla$ denotes the full gradient, $\nabla=\left(\nabla_{x}, \nabla_{a}\right)$. Then there exists some $f \in L^{p}(A)$, such that, as $\epsilon \rightarrow 0$,

$$
f_{B_{\varepsilon}}\|u(x, \cdot)-f\|_{L^{1}(A)} \rightarrow 0
$$

If in addition we assume that $\nabla_{a} f \in L^{p}(A)$, then

$$
v(x, a):= \begin{cases}f(a), & |x| \leq 1, a \in A, \\ u\left(\left(1-\frac{1}{|x|}\right) 2 x, a\right), & 1<|x|<2, a \in A, \\ u(x, a), & 2 \leq|x| \leq 4, a \in A,\end{cases}
$$

is in $W^{1, p}\left(B_{4} \times A\right)$.

Proof. We follow the 4 steps described in the proof of Lemma 1.1,.

Step 1. We claim that

$$
f_{B_{\varepsilon}}\left\|u-\oint_{B_{\varepsilon}} u\right\|_{L^{1}(A)} \leq C \int_{B_{\varepsilon}} \frac{\left\|\nabla_{x} u(x, \cdot)\right\|_{L^{1}(A)}}{|x|^{n-1}}
$$


The proof is the same as the proof of step 1 in Lemma 1.1, except that $|\cdot|$ is replaced by $\|\cdot\|_{L^{1}(A)}$, i.e., we think of $u$ as a function in $W^{1,1}\left(B_{4}, L^{1}(A)\right)$.

Step 2. Both $\lim _{\varepsilon \rightarrow 0} \oint_{\partial B_{\varepsilon}} u(\sigma, \cdot) d \sigma$ and $\lim _{\varepsilon \rightarrow 0} \oint_{B_{\varepsilon}} u(x, \cdot) d x$ exist in $L^{1}(A)$. They are equal, and we denote them by $f$.

Again the proof is the same, replacing $u$ by a vector valued function whose target is the Banach space $L^{1}(A)$.

Step 3. 0 is a Lebesgue point of $u$ considered as a function in $L^{1}\left(B_{4}, L^{1}(A)\right)$, i.e., as $\varepsilon \rightarrow 0$,

$$
\oint_{B_{\varepsilon}}\|u(x, \cdot)-f(\cdot)\|_{L^{1}(A)} d x \rightarrow 0 .
$$

Step 4. $v$ is in $W^{1, p}\left(B_{4} \times A\right)$.

As in the proof of Lemma 1.1, we first obtain

$$
\int_{B_{2} \backslash B_{1}} \int_{A}|v(x, a)| d x d a<\infty,
$$

and

$$
\int_{B_{2} \backslash B_{1}} \int_{A}\left|\nabla_{x} v(x, a)\right|^{p} d x d a \leq C \int_{B_{2}} \int_{A} \frac{\left|\nabla_{x} u(x, a)\right|^{p}}{|x|^{n-1}} d x d a<\infty .
$$

On the other hand, a change of variables yields

$$
\int_{B_{2} \backslash B_{1}} \int_{A}\left|\nabla_{a} v\right|^{p} \leq C \int_{B_{2}} \int_{A} \frac{\left|\nabla_{a} u(x, a)\right|^{p}}{|x|^{n-1}}<\infty .
$$

So far we have proved that $v \in W^{1, p}\left(\left(B_{2} \backslash B_{1}\right) \times A\right)$.

In order to show that $v \in W^{1, p}\left(B_{4} \times A\right)$ we only need to verify on $\partial B_{1} \times A$, in the sense of trace, that $v-f=0$. For $1<r<2$ and $s=2 r-2$, as in the proof of Lemma 1.1,

$$
\int_{\partial B_{r} \times A}|v-f| \leq\left(\frac{2}{s}\right)^{n-1} \int_{\partial B_{s} \times A}|u-f|,
$$

and, because of $(3.7)$,

$$
\frac{1}{s^{n}} \int_{0}^{s}\left\{\int_{\partial B_{\mu} \times A}|u-f|\right\} d \mu \leq \frac{C}{s^{n}} \int_{B_{s} \times A}|u(x, a)-f| \rightarrow 0, \quad \text { as } s \rightarrow 0^{+} .
$$

So, along a subsequence $s_{i} \rightarrow 0^{+}$,

$$
\lim _{i \rightarrow \infty} \int_{\partial B_{r_{i}} \times A}|v-f|=\lim _{i \rightarrow \infty} \frac{1}{s_{i}^{n-1}} \int_{\partial B_{s_{i}} \times A}|u-f|=0
$$

where $r_{i}=\left(s_{1}+2\right) / 2 \rightarrow 1^{+}$. Therefore the trace of $v-f$ on $\left(\partial B_{1}\right) \times A$ is zero. Lemma 3.1 is established. 
Lemma 3.2. Under the hypotheses of Lemma 3.1, set, for $0<t \leq 1$,

$$
u^{t}(x, a):= \begin{cases}f, & |x| \leq t, a \in A, \\ u\left(\left(1-\frac{t}{|x|}\right) 2 x, a\right), & t<|x|<2 t, a \in A, \\ u(x, a), & 2 t \leq|x| \leq 4, a \in A,\end{cases}
$$

and $u^{0}=u$. Then

$$
u^{t} \in C\left([0,1], W^{1, p}\left(B_{4} \times A\right)\right) .
$$

Proof. As a consequence of Lemma $3.1 u^{t}$ is well-defined and is continuous for $t \in(0,1]$. We only need to show that $u^{t} \rightarrow u$ in $W^{1, p}\left(B_{4} \times A\right)$ as $t \rightarrow 0^{+}$. In view of the expression of $u^{t}$, it suffices to prove

$$
\lim _{t \rightarrow 0^{+}}\left\|u^{t}\right\|_{W^{1, p}\left(\left(B_{2 t} \backslash B_{t}\right) \times A\right)}=0 .
$$

This follows from

$$
\sum_{0 \leq|\alpha| \leq 1} \int_{\left(B_{2 t} \backslash B_{t}\right) \times A}\left|\partial^{\alpha} u^{t}\right|^{p} \leq C t^{n-1} \sum_{0 \leq|a| \leq 1} \int_{B_{2 t} \times A} \frac{\left|\partial^{\alpha} u(x, a)\right|^{p}}{|x|^{n-1}} d x d a \rightarrow 0
$$

where we used $\int_{B_{2} \times A} \frac{|u(x, a)|^{p}}{|x|^{n-1}} \leq C \int_{\left(B_{2} \backslash B_{1}\right) \times A}|v(x, a)|^{p}<\infty$. Lemma 3.2 is established.

To prove Proposition 3.2, we need to select a good point $\bar{x}$ so that Lemma 3.2 can be applied, replacing the origin by $\bar{x}$. For this purpose, we need

Lemma 3.3. Let $Y$ be a separable Banach space and $w \in L^{1}\left(B_{4}, Y\right)$. Then for almost all $\bar{x} \in B_{4}$, we have

$$
\frac{1}{\left|B_{\varepsilon}(\bar{x})\right|} \int_{B_{\varepsilon}(\bar{x})}\|w(x)-w(\bar{x})\|_{Y} d x \rightarrow 0 \quad \text { as } \varepsilon \rightarrow 0
$$

Proof. This is well known. For the reader's convenience, we give a sketch. Let $\left\{y_{j}\right\}$ be a dense subset of $Y$, then $\left\|w(x)-y_{j}\right\|_{Y} \in L^{1}\left(B_{4}\right)$. It is well known that for almost all $\bar{x}$ in $B_{4}$

$$
\frac{1}{\left|B_{\varepsilon}(\bar{x})\right|} \int_{B_{\varepsilon}(\bar{x})}\left\|w(x)-y_{j}\right\|_{Y} d x \rightarrow\left\|w(\bar{x})-y_{j}\right\|_{Y}, \quad \text { as } \varepsilon \rightarrow 0 \text {. }
$$

As in [S] (page 11), one can see easily that (3.9) holds for almost all $\bar{x}$ in $B_{4}$.

We now present the 
Proof of Proposition 3.2. Since

$$
\begin{aligned}
& \sum_{0 \leq|\alpha| \leq 1} \int_{B_{4}} \int_{B_{4}} \int_{A} \frac{\left|\partial^{\alpha} u(x, a)\right|^{p}}{|x-\bar{x}|^{n-1}} d x d \bar{x} d a \\
& \leq C \sum_{0 \leq|\alpha| \leq 1} \int_{B_{4}} \int_{A}\left|\partial^{\alpha} u(x, a)\right|^{p} d x d a<\infty,
\end{aligned}
$$

we can pick, in view of Lemma 3.3, a point $\bar{x},|\bar{x}|<1 / 10$, such that

$$
\begin{aligned}
& \int_{B_{4} \times A} \frac{|u(x, a)|^{p}}{|x-\bar{x}|^{n-1}} d x d a+\int_{B_{4} \times A} \frac{|\nabla u(x, a)|^{p}}{|x-\bar{x}|^{n-1}} d x d a<\infty \\
& \frac{1}{\left|B_{\varepsilon}(\bar{x})\right|} \int_{B_{\varepsilon}(\bar{x})}\|u(x, \cdot)-u(\bar{x}, \cdot)\|_{L^{1}(A)} d x \rightarrow 0, \quad \text { as } \varepsilon \rightarrow 0^{+},
\end{aligned}
$$

and

$$
\int_{A}|u(\bar{x}, a)|^{p} d a+\int_{A}\left|\nabla_{a} u(\bar{x}, a)\right|^{p} d a<\infty .
$$

Set, for $0<t \leq 1$.

$$
u^{t}(x, a):= \begin{cases}u(\bar{x}, a), & B_{t / 4}(\bar{x}), a \in A \\ u\left(\left(1-\frac{t}{4|x-\bar{x}|}\right) 2(x-\bar{x}), a\right), & B_{t / 2}(\bar{x}) \backslash B_{t / 4}(\bar{x}), a \in A \\ u(x, a), & x \in B_{4} \backslash B_{t / 2}(\bar{x}),\end{cases}
$$

and $u^{0}=u$. It follows from Lemma 3.2 that $u^{t} \in C\left([0,1], W^{1, p}\left(B_{4} \times A, N\right)\right)$ satisfies $u^{0}=u, u^{t}(x, a)=u(x, a)$ for $|x| \geq 3 / 20$ and all $0 \leq t \leq 1$ and $a \in A$, and $u^{1}(x, a)=u(\bar{x}, a)$ for $|x| \leq 9 / 40$ and all $a \in A$. Proposition 3.2 follows immediately.

\section{Section 4. Proof of Theorem 0.2 When $\operatorname{dim} M \geq 3$}

As before we consider only the case where $\partial M=\emptyset$. We introduce a triangulation $\left\{T_{1}, \cdots, T_{l}\right\}$ of $M$. To simplify the presentation we consider only $\operatorname{dim} M=3$; the passage to higher dimensions is obvious.

Let $\left\{v_{1}, \cdots, v_{k}\right\}$ be the collection of all vertices in the triangulation and let $\left\{e_{1}, \cdots, e_{m}\right\}$ be the collection of all edges (i.e., 1-faces) in the triangulation, $\left\{f_{1}, \cdots, f_{n}\right\}$ be the collection of all the 2 -faces in the triangulation.

In order to connect $u$ to a constant, $Y_{0}$, we proceed step by step. First, we connect $u$ to some $u_{0}$ which equals $Y_{0}$ in some open neighborhood of the vertices $\left\{v_{1} \cdots, u_{k}\right\}$. 
Then, we connect $u_{0}$ to some $u_{1}$ which equals $Y_{0}$ in some open neighborhood of the edges $\left\{e_{1}, \cdots, e_{m}\right\}$.

Next, we connect $u_{1}$ to some $u_{2}$ which equals $Y_{0}$ in some open neighborhood of the 2-faces $\left\{f_{1}, \cdots, f_{n}\right\}$. Finally we connect $u_{2}$ to $Y_{0}$.

Step 0: Connect $u$ to $u_{0}$ which equals $Y_{0}$ near all the vertices.

This is easily done by "opening" of maps (Proposition 1.1) and "connecting" constants (Proposition 1.4).

Step 1: Connect $u_{0}$ to $u_{1}$ which equals $Y_{0}$ near all the edges.

We proceed by induction. First for a single $e_{1}$, recall that $u_{0}$ equals $Y_{0}$ near the two end points of $e_{1}$. By "propagation" (Proposition 1.5) and "bridging" (Proposition 3.1 used with $\ell=0$ requires $p<2$ - it is only for Step 1 that we need $p<2$; for later steps it will suffice to assume $p<3,4$, etc.) we connect $u_{0}$ to $u_{0,1}$ which equals $Y_{0}$ in an open neighborhood of $e_{1} \cup\{$ all vertices $\}$. To proceed with the induction, we may assume that we have connected $u_{0}$ to a map $u_{0, k}$ which equals $Y_{0}$ in an $\varepsilon$-neighborhood of $e_{1} \cup \cdots \cup e_{k} \cup\{$ all vertices . We now wish to add $e_{k+1}$ to the collection. We proceed as in the proof of Cases 1-3 in Section 2. Clearly, there exists $\delta>0$ such that

$$
E \cap\left\{\left\{\delta \text {-neighborhood of } e_{k+1}\right\} \backslash\left\{\frac{\varepsilon}{2} \text {-neighborhood of } \partial e_{k+1}\right\}\right\}=\emptyset,
$$

where $E=e_{1} \cup \cdots \cup e_{k} \cup\{$ all vertices $\}$. By "propagation" and "bridging" we end up with a map which equals $Y_{0}$ near $E \cup e_{k+1}$. We may do so keeping $u_{0, k}$ unchanged outside $\left\{\delta\right.$-neighborhood of $\left.e_{k+1}\right\} \backslash\left\{\frac{\varepsilon}{2}\right.$-neighborhood of $\left.\partial e_{k+1}\right\}$. The resulting map can be taken as $u_{0, k+1}$. This completes the induction and yields a map $u_{1}$ with the required properties.

Step 2: Connect $u_{1}$ to $u_{2}$ which equals $Y_{0}$ near all the 2-faces.

First, for a single 2 -face $f_{1}$, recall that $u_{1}$ equals $Y_{0}$ near $\partial f_{1}$. By Proposition 3.1, applied with $\ell=1$ (this requires only $p<3$ ), we may connect $u_{1}$ to some $u_{1,1}$ which equals $Y_{0}$ near $f_{1} \cup\{$ all edges $\}$. This is done by the same $\varepsilon, \delta$ operation as in Step 1 ; we leave the details to the reader.

Next, we proceed by induction on the number of 2-faces and assume that we have connected $u_{1}$ to a map $u_{1, k}$ which equals $Y_{0}$ in a neighborhood $f_{1} \cup f_{2} \cdots \cup f_{k} \cup\{$ all edges . Now we wish to add another 2-face $f_{k+1}$, to the collection. We argue as in the first step of the induction just above. This completes the induction and yields a map $u_{2}$.

Step 3: Connect $u_{2}$ to $Y_{0}$.

Recall that $u_{2}$ equals $Y_{0}$ near $\partial T_{i}$ for all $1 \leq i \leq l$. Applying Proposition 1.4 ("Filling" a hole) successively on $T_{1}, \cdots, T_{l}$, yields the desired conclusion.

Here we only use $p<3$. 


\section{Section 5. Proof of Theorem 0.3}

Theorem 0.3 can be reformulated as

Theorem 0.3'. Suppose that, for some non-negative integer $k, N$ is $k$-connected, i.e.,

$$
\pi_{0}(N)=\cdots=\pi_{k}(N)=0
$$

and

$$
\operatorname{dim} M \geq k+2, \quad 1 \leq p<k+2 .
$$

Then $W^{1, p}(M, N)$ is path-connected.

We give in this section the proof of Theorem $0.3^{\prime}$. As before we consider only the case where $\partial M=\emptyset$. The proof is by induction on $k$. For $k=0$, this is exactly Theorem 0.2 . Assume that Theorem $0.3^{\prime}$ holds up to $k$, we will prove that it also holds for $k+1$. For $1 \leq p<k+2$, the path-connectedness of $W^{1, p}(M, N)$ follows from the induction hypothesis. So in the following, we assume that

$$
k+2 \leq p<k+3
$$

and wish to prove that any $u \in W^{1, p}(M, N)$ can be connected to a constant.

Let $\left\{T_{1}, \cdots, T_{l}\right\}$ be a triangulation of $M$, and let $\left\{f_{1}, \cdots, f_{m}\right\}$ be all $(k+2)$-cells of the triangulation.

Step 1: Connect $u$ to some $u_{1}$ which equals $Y_{0}$ near $f_{1} \cup \cdots \cup f_{m}$.

We proceed by induction on $m$. First for a single $f_{1}$, we "open" the map $u$ at a "good" point located near $f_{1}$ (Proposition 1.1) and then by "connecting" constants (Proposition 1.3) and "propagation" of constants (Proposition 1.4) we connect $u$ to some $u_{0,1}$ which equals $Y_{0}$ near $f_{1}$. To proceed with the induction, we may assume that we have connected $u$ to some $u_{0, j}$ which equals $Y_{0}$ near $f_{1} \cup \cdots \cup f_{j}$. Let $E=f_{j+1} \cap\left(f_{1} \cup \cdots \cup f_{j}\right)$. If $E=\emptyset$, then, in the same way as we have connected $u$ to $u_{0,1}$, we can connect $u$ to some $u_{0, j+1}$ which equals $Y_{0}$ near $f_{1} \cup \cdots \cup f_{j+1}$. This can be achieved without changing the values of $u$ near $f_{1} \cup \cdots \cup f_{j}$. If $E \neq \emptyset$, recall that $u_{0, j}=Y_{0}$ in the $\epsilon$-neighborhood of $E$ for some $\epsilon>0$. The value of $\epsilon$ will be taken small enough so that the following arguments can go through. Let $B_{\epsilon}$ be the ball of radius $\epsilon$ in $\mathbb{R}^{\operatorname{dim} M-k-1}$ centered at the origin, and let

$$
\varphi: B_{\epsilon} \times \mathbb{S}^{k+1} \rightarrow M
$$

be a diffeomorphism such that for any $(x, \sigma) \in \partial B_{\epsilon} \times \mathbb{S}^{k+1},\{\varphi(s x, \sigma) ; 0<s<1\}$ is a geodesic parameterized by arclength $s$; moreover,

$$
\partial f_{j+1} \subset \varphi\left(B_{\epsilon^{4}} \times \mathbb{S}^{k+1}\right), \quad \varphi\left(\{0\} \times \mathbb{S}^{k+1}\right) \subset f_{j+1} .
$$


[ Notations would be much simpler if we could let $\varphi\left(\{0\} \times \mathbb{S}^{k+1}\right)=\partial f_{j+1}$. But such $\varphi$ would not be smooth. What we have done above is to select a smooth $\varphi$ such that $\varphi\left(\{0\} \times \mathbb{S}^{k+1}\right)$ is as close to $\partial f_{j+1}$ as we wish.]

Consider the composition

$$
v=u \circ \varphi: B_{5 \epsilon^{2}} \times \mathbb{S}^{k+1} \rightarrow N .
$$

By Proposition 3.2 (see also Remark 3.3) we can connect $v$ to $\tilde{v}$ in $W^{1, p}\left(B_{5 \epsilon^{2}} \times \mathbb{S}^{k+1}, N\right)$ such that

$$
\begin{gathered}
\tilde{v}(x, \sigma)=v(x, \sigma), \quad \forall x \in B_{5 \epsilon^{2}} \backslash B_{4 \epsilon^{2}}, \forall \sigma, \\
\tilde{v}(x, \sigma)=V(\sigma), \quad \forall x \in B_{3 \epsilon^{2}}, \forall \sigma,
\end{gathered}
$$

for some $V \in W^{1, p}\left(\mathbb{S}^{k+1}, N\right)$. Moreover,

$$
\tilde{u}(P):= \begin{cases}u(P), & P \in M \backslash \varphi\left(B_{5 \epsilon^{2}} \times \mathbb{S}^{k+1}\right), \\ \tilde{v} \circ \varphi^{-1}(P), & P \in \varphi\left(B_{5 \epsilon^{2}} \times \mathbb{S}^{k+1}\right)\end{cases}
$$

has the property

$$
\tilde{u}=Y_{0} \text { in the } \frac{\epsilon}{2}-\text { neighborhood of } E \text {. }
$$

So we have connected $u$ to $\tilde{u}$, which is still $Y_{0}$ in the $\frac{\epsilon}{2}$-neighborhood of $f_{1} \cup \cdots \cup f_{j}$.

Choose disjoint open sets $O_{1}, \cdots, O_{l} \subset \mathbb{S}^{k+1}$ such that each $O_{i}$ is diffeomorphic to a unit ball in $\mathbb{R}^{k+1}$, and

$$
\begin{gathered}
E \subset \varphi\left(B_{9 \epsilon^{3}} \times \cup_{i=1}^{l} O_{i}\right) \subset \frac{\epsilon}{2}-\text { neighborhood of } E, \\
\operatorname{dist}\left(\varphi\left(B_{\epsilon^{3}} \times \cap_{i=1}^{l}\left(\mathbb{S}^{k+1} \backslash O_{i}\right)\right), E\right)>\epsilon^{2} .
\end{gathered}
$$

Since $p \geq k+2$, we know from the Sobolev embedding theorem that $V \in W^{1, p}\left(\mathbb{S}^{k+1}, N\right) \subset C^{0}\left(\mathbb{S}^{k+1}, N\right)$. Therefore, by a homotopy, we may assume that $V \in C^{\infty}\left(\mathbb{S}^{k+1}, N\right)$ and

$$
\tilde{v}(x, \sigma)=V(\sigma), \quad x \in B_{2 \epsilon^{2}} .
$$

Indeed this can be achieved as follows. Let $0<\delta<<\epsilon^{4}$ and let $\eta \in C_{c}^{\infty}\left(B_{3 \epsilon^{2}}\right)$ satisfying $0 \leq \eta \leq 1, \eta(x)=1$ for $x \in B_{2 \epsilon^{2}}, \eta(x)=0$ for $x \in B_{3 \epsilon^{2}} \backslash B_{\frac{5}{2} \epsilon^{2}}$. Set

$$
\tilde{V}^{t}(x, \sigma)=P\left\{\int V(\sigma-t \delta \eta(x) y) \rho(y) d y\right\},
$$


where $\rho(y)$ is the usual mollifier and $P$ is the projection to $N$. Here we have abused the notation since the integration should be done on $\mathbb{S}^{k+1}$ instead of on Euclidean space as the notation suggests. Since $V$ is continuous, for $\delta$ small enough, $\widetilde{V}^{t}$ is $C^{\infty}$ in $t, x$, and $\sigma$. Therefore $V=\widetilde{V}^{0}$ has been connected to $\widetilde{V}^{1}$ which has the desired properties.

It is not difficult to deduce from (5.4) and (5.6) that

$$
V=Y_{0} \quad \text { on } \cup_{i=1}^{l} O_{i}
$$

Since $N$ is $(k+1)$-connected, there exists $V^{t} \in C^{\infty}\left([0,1] \times \mathbb{S}^{k+1}, N\right)$ such that

$$
\begin{gathered}
V^{t}(\sigma)=Y_{0}, \quad \forall 0 \leq t \leq 1, \sigma \in \cup_{i=1}^{l} O_{i}, \\
V^{0}=V \\
V^{1}=Y_{0} .
\end{gathered}
$$

The existence of a continuous homotopy satisfying (5.10)-(5.12) follows from standard results in topology (e.g., Corollary 6.19, page 244 in [Wh], applied with $X$ being $\mathbb{S}^{k+1}$ quotient the union of the $O_{j}^{\prime}$ s), while the existence of a $C^{\infty}$ homotopy $V^{t}$ can be achieved by some standard arguments using mollifiers.

Let $\rho \in C^{\infty}\left(B_{9 \epsilon^{3}}\right)$ be such that $0 \leq \rho \leq 1, \rho(x)=1$ for $x \in B_{2 \epsilon^{3}}, \rho(x)=0$ for $x \in B_{9 \epsilon^{3}} \backslash B_{4 \epsilon^{3}}$. We set, for $0 \leq t \leq 1$,

$$
\tilde{v}^{t}(x, \sigma)=V^{t \rho(x)}(\sigma), \quad(x, \sigma) \in B_{9 \epsilon^{3}} \times \mathbb{S}^{k+1} .
$$

Clearly this is an admissible homotopy and

$$
\begin{gathered}
\tilde{v}^{0}(x, \sigma)=\tilde{v}(x, \sigma), \quad(x, \sigma) \in B_{9 \epsilon^{3}} \times \mathbb{S}^{k+1}, \\
\tilde{v}^{1}(x, \sigma)=Y_{0}, \quad(x, \sigma) \in B_{2 \epsilon^{3}} \times \mathbb{S}^{k+1} .
\end{gathered}
$$

By defining, for $0 \leq t \leq 1$,

$$
w^{t}(P):= \begin{cases}\tilde{u}(P), & P \in M \backslash \varphi\left(B_{9 \epsilon^{3}} \times \mathbb{S}^{k+1}\right), \\ \tilde{v}^{t} \circ \varphi^{-1}(P), & P \in \varphi\left(B_{9 \epsilon^{3}} \times \mathbb{S}^{k+1}\right),\end{cases}
$$

we connect $\tilde{u}\left(=w^{0}\right)$ to $w^{1}$. According to the definition,

$$
w^{1}(P)=Y_{0} \quad \forall P \in \varphi\left(B_{2 \epsilon^{3}} \times \mathbb{S}^{k+1}\right),
$$


which implies, in view of (5.3), that $w^{1}=Y_{0}$ near $\partial f_{j+1}$. As mentioned earlier, the value of $\epsilon$ has been taken very small and therefore (using in particular (5.7)) along all the homotopies we have made the values in some open neighborhood of $f_{1} \cup \cdots \cup f_{j}$ have been preserved as $Y_{0}$.

Finally we apply Proposition 3.1 (with $\ell=k+1$ and $n=\operatorname{dim} M$ ) to connect $w^{1}$ to some $u_{0, j+1}$ which equals $Y_{0}$ near $f_{1} \cup \cdots \cup f_{j+1}$. We have completed Step 1 .

Step 2. Connect $u_{1}$ to $Y_{0}$.

If $\operatorname{dim} M=k+3$, we already know from Step 1 that $u_{1}=Y_{0}$ near $\partial T_{1} \cup \cdots \cup \partial T_{l}$. Applying the technique of "filling" a hole (Proposition 1.3) successively to $T_{1}, \cdots, T_{l}$, we connect $u_{1}$ to $Y_{0}$. If $\operatorname{dim} M>k+3$, let $\left\{e_{1}, \cdots, e_{i}\right\}$ be all $(k+3)$-cells of the triangulation and we know from Step 1 that $u_{1}=Y_{0}$ near $\partial e_{1} \cup \cdots \cup \partial e_{i}$. Applying Proposition 3.1 (with $\ell=k+2$ and $n=\operatorname{dim} M$ ) successively to $e_{1}, \cdots, e_{i}$, we connect $u_{1}$ to some $u_{2}$ which equals $Y_{0}$ near $e_{1} \cup \cdots \cup e_{i}$. Continuing in this way (by induction), we connect $u_{2}$ to some $u_{\text {dim } M-k-2}$ which equals $Y_{0}$ near $\partial T_{1} \cup \cdots \cup \partial T_{l}$. Finally, by the technique of "filling" a hole, we connect $u_{\operatorname{dim} M-k-2}$ to $Y_{0}$. This completes Step 2.

We have verified that Theorem $0.3^{\prime}$ holds for $k+1$ as well. The proof of Theorem $0.3^{\prime}$ is complete.

\section{Section 6. Evidence in support of Conjecture 1: Proof of Theorem 0.4}

Recall the statement of Conjecture 1.

Conjecture 1. Given $u \in W^{1, p}(M, N)$ (any $1 \leq p<\infty$, any $M$, any $N$ ), there exists a $v \in C^{\infty}(M, N)$ and a path $u^{t} \in C\left([0,1], W^{1, p}(M, N)\right)$ such that $u^{0}=u$ and $u^{1}=v$.

In this section we prove the following special case of Conjecture 1.

Theorem 0.4. If $\operatorname{dim} M=3$ and $\partial M \neq \emptyset$ (any $N$ and any $p$ ), Conjecture 1 holds.

The proof of Theorem 0.4 relies on the following

Proposition 6.1. Let $M$ and $N$ be smooth connected compact ${ }^{2}$ oriented Riemannian manifold with or without boundary. Assume $\operatorname{dim} M=3$ and $p \geq 1$. Then for every $u \in W^{1, p}(M, N)$, there exists a continuous path in $W^{1, p}(M, N)$ connecting $u$ to some $v$ which is $C^{\infty}$ except possibly at one point.

Proof of Proposition 6.1. Let $u \in W^{1, p}(M, N)$. If $p>3$, then $u \in C^{0}(M, N)$ by the Sobolev embedding theorem and we can actually take $v$ to be $C^{\infty}$ everywhere. If $p=3$, then $W^{1, p}(M, N) \subset V M O$ and we can also take $v$ to be $C^{\infty}$ everywhere (see the Appendix). On the other hand, if $p<2$, then by Theorem 0.2 we can actually take $v$ to be a constant map. So in the following we assume that

$$
2 \leq p<3
$$

\footnotetext{
${ }^{2}$ See Remark A.1 in the Appendix if $N$ is not compact.
} 
As before we only consider the case where $\partial M=\emptyset$. We introduce a triangulation of $M$, denoted by $\left\{T_{1}, \cdots, T_{l}\right\}$. We divide the proof into three steps. First, we connect $u$ to some $u_{1}$ which is $W^{1, p}(M, N) \cap \operatorname{Lip}$ near $\partial T_{1} \cup \cdots \cup \partial T_{l}$. Next, we connect $u_{1}$ to some $u_{2}$ which is $W^{1, p}(M, N) \cap$ Lip except possibly at finite points. Finally, we connect $u_{2}$ to some $w$ which is $W^{1, p}(M, N) \cap$ Lip except possibly at one point. Here Lip means Lipschitz.

Step 1. Connect $u$ to some $u_{1}$ which is $W^{1, p}(M, N) \cap \operatorname{Lip}\left(\right.$ near $\partial T_{1} \cup \cdots \cup \partial T_{l}$ ).

We proceed by induction on $l$. By "opening" $u$ at a "good" point in $T_{1}$ (Proposition 1.1) and "propagating" the constant (Proposition 1.5), we may connect $u$ to some $u_{0,1}$ which is constant near $T_{1}$. We assume that we have connected $u$ to some $u_{0, k}$ which is $W^{1, p}(M, N) \cap \operatorname{Lip}$ (near $\partial T_{1} \cup \cdots \cup \partial T_{k}$ ), and we wish to add $\partial T_{k+1}$ to the collection. Let $E=\partial T_{k+1} \cap\left(\partial T_{1} \cup \cdots \cup \partial T_{k}\right)$. If $E=\emptyset$, then, in the same way as we have connected $u$ to $u_{0,1}$, we easily connect $u_{0, k}$ to some $u_{0, k+1}$ which is $W^{1, p}(M, N) \cap C^{0}$ (near $\partial T_{1} \cup \cdots \cup$ $\left.\partial T_{k+1}\right)$. If $E \neq \emptyset$, recall that $u_{0, k}$ is $W^{1, p}(M, N) \cap \operatorname{Lip}$ in the $\epsilon$-neighborhood of $E$ for some $\epsilon>0$. The value of $\epsilon$ will be taken small enough so that the following arguments can go through. Let $B_{\epsilon}=(-\epsilon, \epsilon)$ and let

$$
\varphi: B_{\epsilon} \times \mathbb{S}^{2} \rightarrow M
$$

be a diffeomorphism such that for any $(x, \sigma) \in \partial B_{\epsilon} \times \mathbb{S}^{2},\{\varphi(s x, \sigma) ; 0<s<1\}$ is a geodesic parameterized by arclength $s$; moreover,

$$
\partial T_{k+1} \subset \varphi\left(B_{\epsilon^{4}} \times \mathbb{S}^{2}\right), \quad \varphi\left(\{0\} \times \mathbb{S}^{2}\right) \subset T_{k+1}
$$

Consider the composition

$$
v=u \circ \varphi: B_{5 \epsilon^{2}} \times \mathbb{S}^{2} \rightarrow N .
$$

By Proposition 3.2 (see also Remark 3.3) we can connect $v$ to $\tilde{v}$ in $W^{1, p}\left(B_{5 \epsilon^{2}} \times \mathbb{S}^{2}, N\right)$ such that

$$
\begin{gathered}
\tilde{v}(x, \sigma)=v(x, \sigma), \quad \forall x \in B_{5 \epsilon^{2}} \backslash B_{4 \epsilon^{2}}, \forall \sigma, \\
\tilde{v}(x, \sigma)=V(\sigma), \quad \forall x \in B_{3 \epsilon^{2}}, \forall \sigma,
\end{gathered}
$$

for some $V \in W^{1, p}\left(\mathbb{S}^{2}, N\right)$. Moreover,

$$
\tilde{u}(P):= \begin{cases}u(P), & P \in M \backslash \varphi\left(B_{5 \epsilon^{2}} \times \mathbb{S}^{2}\right) \\ \tilde{v} \circ \varphi^{-1}(P), & P \in \varphi\left(B_{5 \epsilon^{2}} \times \mathbb{S}^{2}\right)\end{cases}
$$

has the property that

$$
\tilde{u} \text { is } W^{1, p}(M, N) \cap \operatorname{Lip} \text { in the } \frac{\epsilon}{2}-\text { neighborhood of } E \text {. }
$$


So we have connected $u$ to $\tilde{u}$, which is still $W^{1, p}(M, N) \cap \operatorname{Lip}$ in the $\frac{\epsilon}{2}$-neighborhood of $\partial T_{1} \cup \cdots \cup \partial T_{k}$. Since $W^{1, p}\left(\mathbb{S}^{2}, N\right) \subset V M O$ (here we use $p \geq 2$; in fact if $p>2$, $\left.W^{1, p} \subset C^{0}\right)$, we may assume, after making a homotopy, that $V \in C^{\infty}\left(\mathbb{S}^{2}, N\right)$ and

$$
\tilde{v}(x, \sigma)=V(\sigma), \quad x \in B_{2 \epsilon^{2}}
$$

Indeed this can be achieved by the same argument as the one following formula (5.8). Step 1 is complete.

Step 2. Connect $u_{1}$ to some $u_{2}$ which is $W^{1, p}(M, N) \cap$ Lip except possibly at finite points.

This step can be easily deduced by applying the following lemma successively on $T_{1}, \cdots, T_{l}$.

Let $B_{1}$ denote the unit ball of $\mathbb{R}^{3}$ centered at the origin and let $1 \leq p<3$. Assume that $u \in W^{1, p}\left(B_{1}\right)$ and $u$ is Lip near $\partial B_{1}$. Define, for $0<t \leq 1$,

$$
u^{t}(x)=\tilde{u}\left(\frac{x}{t}\right), \quad x \in B_{1}
$$

where

$$
\tilde{u}(x)= \begin{cases}u(x), & x \in B_{1}, \\ u\left(\frac{x}{|x|}\right), & x \in \mathbb{R}^{3} \backslash B_{1}\end{cases}
$$

and

$$
u^{0}(x)=u\left(\frac{x}{|x|}\right), \quad x \in B_{1} \backslash\{0\}
$$

Lemma 6.1. $u^{t} \in C\left([0,1], W^{1, p}\left(B_{1}\right)\right)$.

Proof. It is elementary.

Step 3. Connect $u_{2}$ to some $w$ which is $W^{1, p}(M, N) \cap$ Lip except possibly at one point.

Since $u_{2}$ has at most finitely many singular points and $M$ is connected, we can easily connect $u_{2}$ to some $u_{2,1}$ which is $W^{1, p}(M, N) \cap$ Lip away from a small geodesic ball, say $B_{\epsilon}(\bar{P})$ (it suffices to fix a singular point as $\bar{P}$ and to move smoothly the other singular points close to $\bar{P})$. Applying Lemma 6.1 to $B_{2 \epsilon}(\bar{P})$, we connect $u_{2,1}$ to some $w \in W^{1, p}(M, N) \cap$ $\operatorname{Lip}(M \backslash\{\bar{P}\}, N)$. By Proposition A.4, we connect $w$ to some $v \in W^{1, p}(M, N) \cap C^{\infty}(M \backslash$ $\{\bar{P}\}, N)$.

Proof of Theorem 0.4. Let $\nu(Q)$ denote the unit inner normal at $Q \in \partial M$. For some $\epsilon>0$,

$$
\varphi(Q, s):=\exp _{Q}(s \nu(Q))
$$


is a diffeomorphism from $\partial M \times[0,3 \epsilon]$ to a neighborhood of $\partial M$, where $\exp _{Q}(s \nu(Q))$ is the exponential map.

By Proposition 6.1 we can connect $u$ to some $u_{1}$ which is $C^{\infty}$ except possibly at one point. Since $M$ is connected, we easily connect $u_{1}$ to some $u_{2} \in C^{\infty}(M \backslash\{\bar{P}\}, N)$ with $\operatorname{dist}(\bar{P}, \partial M)<\epsilon$. This singularity can be removed through a homotopy by pushing $\partial M$ into $M$ along the normal. Indeed, let $\rho \in C^{\infty}(\mathbb{R}),-1<\rho^{\prime} \leq 0, \rho(\tau)=1$ if $\tau<1 ; \rho(\tau)=0$ if $\tau>3$. Define for $0 \leq t \leq 1$,

$$
u_{2}^{t}(P):= \begin{cases}u_{2}\left(Q, s+t \epsilon \rho\left(\frac{s}{\epsilon}\right)\right), & P=\varphi(Q, s),(Q, s) \in \partial M \times[0,3 \epsilon], \\ u_{2}(P), & P \in M \backslash \varphi(\partial M \times[0,3 \epsilon]) .\end{cases}
$$

This homotopy connects $u_{2}\left(=u_{2}^{0}\right)$ to $u_{2}^{1} \in C^{\infty}(M, N)$.

\section{Section 7. Everything you Wanted to Know about $W^{1, p}\left(M, \mathbb{S}^{1}\right)$}

The main result of this section is the following special case of Conjecture 1.

Theorem 0.5. If $N=\mathbb{S}^{1}$ (any $M$ and any $p$ ), Conjecture 1 holds.

We start with some preliminaries which will be used in the proof. For $n \geq n^{\prime} \geq 1$, we write $\mathbb{R}^{n}=\mathbb{R}^{n^{\prime}} \times \mathbb{R}^{n-n^{\prime}}$ and $x \in \mathbb{R}^{n}$ as $x=\left(x^{\prime}, x^{\prime \prime}\right) \in \mathbb{R}^{n^{\prime}} \times \mathbb{R}^{n-n^{\prime}}$. Let

$$
D^{\prime}=\left\{x^{\prime} \in \mathbb{R}^{n^{\prime}} ;\left|x^{\prime}\right|<1\right\} \quad \text { and } \quad D^{\prime \prime}=\left\{x^{\prime \prime} \in \mathbb{R}^{n-n^{\prime}} ;\left|x^{\prime \prime}\right|<1\right\}
$$

be the unit balls in $\mathbb{R}^{n^{\prime}}$ and $\mathbb{R}^{n-n^{\prime}}$ respectively.

Lemma 7.1. For $n \geq n^{\prime} \geq 1$ and $p \geq 2$, let $f^{0}, f^{1} \in W^{1, p}\left(D^{\prime}, \mathbb{S}^{1}\right)$ with

$$
f^{0}=f^{1} \quad \text { on } \partial D^{\prime} \text {. }
$$

Then there exists $F^{t} \in C\left([0,1], W^{1, p}\left(D^{\prime} \times D^{\prime \prime}, \mathbb{S}^{1}\right)\right)$ such that

$$
\begin{gathered}
F^{0}\left(x^{\prime}, x^{\prime \prime}\right)=f^{0}\left(x^{\prime}\right) \quad \text { on } D^{\prime} \times D^{\prime \prime}, \\
F^{t}\left(x^{\prime}, x^{\prime \prime}\right)=f^{0}\left(x^{\prime}\right) \quad \forall 0 \leq t \leq 1,\left|x^{\prime \prime}\right|>\frac{9}{10}, x^{\prime} \in D^{\prime}, \\
F^{t}\left(x^{\prime}, x^{\prime \prime}\right)=f^{0}\left(x^{\prime}\right)=f^{1}\left(x^{\prime}\right), \quad \forall 0 \leq t \leq 1, x^{\prime} \in \partial D^{\prime}, x^{\prime \prime} \in D^{\prime \prime}, \\
F^{1}\left(x^{\prime}, x^{\prime \prime}\right)=f^{1}\left(x^{\prime}\right), \quad \forall\left|x^{\prime \prime}\right|<\frac{1}{10}, x^{\prime} \in D^{\prime} .
\end{gathered}
$$

Moreover if both $f^{0}$ and $f^{1}$ are smooth in some open set $O^{\prime}$ in $D^{\prime}$, then $F^{t}$ is smooth in $O^{\prime} \times D^{\prime \prime}$.

Proof. Since $p \geq 2$, it follows from Bethuel and Zheng [BZ] (see also Bourgain, Brezis and Mironescu $[\mathrm{BBM}])$ that there exists $h^{0}, h^{1} \in W^{1, p}\left(D^{\prime}, \mathbb{R}\right)$ such that

$$
f^{0}=e^{i h^{0}} \quad \text { and } \quad f^{1}=e^{i h^{1}} .
$$


Set

$$
f^{t}=e^{i t h^{1}+i(1-t) h^{0}}, \quad 0 \leq t \leq 1 .
$$

Consider a smooth cut-off function $\rho \in C^{\infty}(\mathbb{R}), 0 \leq \rho \leq 1, \rho(s)=1$ for $|s| \leq 1 / 10$, and $\rho(s)=0$ for $|s| \geq 9 / 10$. Define

$$
F^{t}=f^{t \rho\left(\left|x^{\prime \prime}\right|\right)}\left(x^{\prime}\right), \quad 0 \leq t \leq 1
$$

It is easy to see that $F^{t}$ satisfies the desired properties.

We also need a variant of Proposition 3.2. For $\epsilon>0$, let

$$
A_{\epsilon}=\{a \in A ; \operatorname{dist}(a, \partial A)>\epsilon\} .
$$

Proposition 7.1. Let $A$ be a smooth compact Riemannian manifold with boundary, Nbe a smooth Riemannian manifold with or without boundary, and let $u \in W^{1, p}\left(B_{4} \times A, N\right)$ where $p \geq 1$ and $B_{4}$ is the ball in $\mathbb{R}^{n}$ of radius 4 and centered at the origin. Then for all $\epsilon>0$, there exists a continuous path $u^{t} \in C\left([0,1], W^{1, p}\left(B_{4} \times A, N\right)\right)$ such that $u^{0}=u$,

$$
u^{t}(x, a)=u(x, a), \quad(x, a) \in\left(B_{4} \times A\right) \backslash\left(B_{2 / 3} \times A_{\epsilon}\right), 0 \leq t \leq 1,
$$

and for some $Y \in W^{1, p}(A, N)$,

$$
u^{1}(x, a)=Y(a) \quad x \in B_{1 / 3}, a \in A_{2 \epsilon} .
$$

Moreover, if for some $\delta>0, u$ is Lip in $B_{4} \times\left(A \backslash A_{2 \delta}\right)$, then $u^{t}$ can be taken to satisfy in addition $u^{1} \in \operatorname{Lip}\left(B_{4} \times\left(A \backslash A_{\delta}\right), N\right)$.

The proof of Proposition 7.1 is a variant of the proof of Proposition 3.2. We point out one modification, since the others are more obvious. What we will need is a variant of Lemma 3.2. Let $\rho \in C^{\infty}(A), 0 \leq \rho \leq 1, \rho(a)=1$ for $a \in A_{2 \epsilon}, \rho(a)=0$ for $a \in A \backslash A_{\epsilon}$.

Lemma 7.2. Under the hypotheses of Lemma 3.1, set, for $0<t \leq 1$,

$$
u^{t}(x, a):= \begin{cases}f, & |x| \leq t \rho(a), a \in A, \\ u\left(\left(1-\frac{t \rho(a)}{|x|}\right) 2 x, a\right), & t \rho(a)<|x|<2 t \rho(a), a \in A, \\ u(x, a), & 2 t \rho(a) \leq|x| \leq 4, a \in A,\end{cases}
$$

and $u^{0}=u$. Then

$$
u^{t} \in C\left([0,1], W^{1, p}\left(B_{4} \times A\right)\right) .
$$

The proof of Lemma 7.2 is a modification of the proof of Lemma 3.2 (and the statement of Lemma 3.1 and its proof). We leave the details to the reader. 
Proof of Theorem 0.5. Let $n=\operatorname{dim} M$. If $1 \leq p<2$, the conclusion follows from Theorem 0.2 . On the other hand, if $p \geq n$, the conclusion follows from Proposition A.2. So we only need to consider the case

$$
n \geq 3 \quad \text { and } \quad 2 \leq p<n
$$

As always, we discuss only the case where $\partial M=\emptyset$. Let $\left\{T_{1}, \cdots, T_{l}\right\}$ be a triangulation of $M$. We will first connect $u$ to some $u_{1}$ which is Lip near all $[p]$-cells of the triangulation. Then, by induction on the dimensions of cells $([p]$-cells, $([p]+1)$-cells, $\cdots,(n-1)$-cells $)$, we connect $u_{1}$ to some $\tilde{u}_{2}$ which is Lip near $\partial T_{1} \cup \cdots \cup \partial T_{l}$, and then connect this $\tilde{u}_{2}$ to some $u_{2}$ which is $C^{\infty}$ near $\partial T_{1} \cup \cdots \cup \partial T_{l}$. Finally we connect $u_{2}$ to some $v \in C^{\infty}(M, N)$.

Step 1. Connect $u$ to some $u_{1}$ which is Lip near all $[p]$-cells.

Let $\left\{e_{1}, \cdots, e_{m}\right\}$ denote all the $([p]+1)$-cells. We proceed by induction. As usual, by "opening" at a "good" point located near $e_{1}$ and "propagating" the constant, we connect $u$ to some $u_{0,1}$ which is constant near $e_{1}$. Assume that we have connected $u$ to some $u_{0, k}$ which is Lip near $\partial e_{1} \cup \cdots \cup \partial e_{k}$, we wish to add $\partial e_{k+1}$ to the collection. Set $E=\partial e_{k+1} \cap\left(\partial e_{1} \cup \cdots \cup \partial e_{k}\right)$. If $E=\emptyset$, we easily connect $u_{0, k}$ to some $u_{0, k+1}$ which is Lip near $\partial e_{1} \cup \cdots \cup \partial e_{k+1}$. If $E \neq \emptyset$, recall that $u_{0, k}$ is Lip in the $\epsilon$-neighborhood of $E$ for some $\epsilon>0$. The value of $\epsilon$ will be taken small enough so that the following arguments can go through. Let $B_{\epsilon}$ be the ball of radius $\epsilon$ in $\mathbb{R}^{n-[p]}$ centered at the origin, and let

$$
\varphi: B_{\epsilon} \times \mathbb{S}^{[p]} \rightarrow M
$$

be a diffeomorphism such that for any $(x, y) \in \partial B_{\epsilon} \times \mathbb{S}^{[p]},\{\varphi(s x, y) ; 0<s<1\}$ is a geodesic parameterized by arclength $s$; moreover,

$$
\partial e_{k+1} \subset \varphi\left(B_{\epsilon^{4}} \times \mathbb{S}^{[p]}\right), \quad \varphi\left(\{0\} \times \mathbb{S}^{[p]}\right) \subset e_{k+1} .
$$

By "opening" techniques, as in Step 1 of the proof of Theorem 0.3 , we may connect $u_{0, k}$ to some $u_{0, k+1}$ which is Lip near $\partial e_{1} \cup \cdots \cup \partial e_{k+1}$. This completes the induction and yields a map $u_{1}$ with the desired property.

Step 2. Connect $u_{1}$ to some $u_{2}$ which is $C^{\infty}$ near $\partial T_{1} \cup \cdots \cup \partial T_{l}$.

If $n-1=[p]$, this step is already achieved in Step 1. Otherwise

$$
n \geq[p]+2
$$

We will only show how to connect $u_{1}$ to some $w$ which is Lip near all $([p]+1)$-cells since the remaining can be established, by induction on the dimensions of cells, using the same arguments. 
Let $\left\{e_{1}, \cdots, e_{m}\right\}$ denote all the $([p]+1)$-cells. We will first connect $u_{1}$ to some $\xi$ which is Lip near $e_{1} \cup\{$ all $[p]-$ cells $\}$.

We know that $u_{1}$ is Lip in the $\epsilon$-neighborhood of $\partial e_{1} \cup \cdots \partial e_{m}$ for some $\epsilon>0$. The value of $\epsilon$ will be taken small enough so that the following arguments go through. Let $B_{\epsilon}$ be the ball of radius $\epsilon$ in $\mathbb{R}^{n-[p]-1}$ centered at the origin and $D$ be a unit disk in $\mathbb{R}^{[p]+1}$, and let

$$
\varphi: B_{\epsilon} \times D \rightarrow M
$$

be a diffeomorphism such that for $(x, y) \in \partial B_{\epsilon} \times D,\{\varphi(s x, y) ; 0<s<1\}$ is a geodesic parameterized by arclength $s$; moreover,

$$
\begin{gathered}
e_{1} \subset \varphi\left(B_{\epsilon^{4}} \times D\right) \subset 2 \epsilon^{4}-\text { neighborhood of } e_{1}, \\
\partial e_{1} \subset \varphi\left(B_{\epsilon^{4}} \times \partial D\right) \subset 2 \epsilon^{4}-\text { neighborhood of } \partial e_{1} .
\end{gathered}
$$

Let $D^{\prime} \subset D$ be a slightly smaller disk such that

$$
\begin{gathered}
e_{1} \subset \epsilon^{4}-\text { neighborhood of } \varphi\left(B_{\epsilon^{4}} \times D^{\prime}\right) \subset 4 \epsilon^{4}-\text { neighborhood of } e_{1}, \\
\partial e_{1} \subset \epsilon^{4}-\text { neighborhood of } \varphi\left(B_{\epsilon^{4}} \times \partial D^{\prime}\right) \subset 4 \epsilon^{4}-\text { neighborhood of } \partial e_{1} .
\end{gathered}
$$

Applying Proposition 7.1 to $u_{1} \circ \varphi$ (modulo another diffeomorphism to change the radius of balls, etc.), we connect $u_{1}$ to some $u_{1,1}$ which has the following properties:

$$
\begin{gathered}
u_{1,1} \text { is Lip in the } \frac{\epsilon}{2}-\text { neighborhood of } \partial e_{1} \cup \cdots \partial e_{m}, \\
u_{1,1} \circ \varphi(x, y)=V(y) \quad \forall(x, y) \in B_{3 \epsilon^{2}} \times D^{\prime},
\end{gathered}
$$

where $V \in W^{1, p}\left(D^{\prime}, \mathbb{S}^{1}\right)$ and $V$ is Lip near $\partial D^{\prime}$. But $\partial D^{\prime}$ is a $[p]$-sphere and, since $[p]>1$, $\pi_{[p]}\left(\mathbb{S}^{1}\right)=0$, we can pick $f^{1} \in \operatorname{Lip}\left(\overline{D^{\prime}}, \mathbb{S}^{1}\right)$ with

$$
f^{1}=V \quad \text { on } \partial D^{\prime}
$$

Applying Lemma 7.1 (change the radius of balls, etc.) with $D^{\prime \prime}=B_{3 \epsilon^{2}}, n^{\prime}=[p]+1, f^{0}=V$, we connect $u_{1,1}$ to some $\xi$ which is Lip near $e_{1} \cup\left\{\partial e_{2} \cup \cdots \partial e_{m}\right\}=e_{1} \cup\{$ all $[p]$ - cells $\}$.

Doing the same successively on $e_{2}, \cdots, e_{m}$ we connect $u_{1}$ to some $w$ which is Lip near all $([p]+1)$-cells.

Next we show by the same argument that we can connect $w$ (already Lip near all $([p]+1)$-cells $)$ to some map which is Lip near all $([p]+2)$-cells. Eventually (by induction), we connect $u_{1}$ to some $\tilde{u}_{2}$ which is Lip near $\partial T_{1} \cup \cdots \cup \partial T_{l}$, and then, by some mollifier argument (Proposition A.5 in the Appendix), connect this $\tilde{u}_{2}$ to some $u_{2}$ which is $C^{\infty}$ near $\partial T_{1} \cup \cdots \cup \partial T_{l}$. 
Step 3. Connect $u_{2}$ to some $v \in C^{\infty}\left(M, \mathbb{S}^{1}\right)$.

Let $B$ be a unit ball in $\mathbb{R}^{n}$ and let

$$
\varphi: B \rightarrow T_{1}
$$

be a diffeomorphism onto $\varphi(B)$ such that $u_{2}$ is $C^{\infty}$ in $\overline{T_{1} \backslash \varphi(B)}$. So $u_{2} \circ \varphi$ is $C^{\infty}$ on $\partial B$. Since $\pi_{n-1}\left(\mathbb{S}^{1}\right)=0(n \geq 3)$, we can pick $f^{1} \in C^{\infty}\left(B, \mathbb{S}^{1}\right)$ such that $f^{1}=u_{2} \circ \varphi$ on $\partial B$. Applying Lemma 7.1 with $n^{\prime}=n, f^{0}=u_{2} \circ \varphi$, we connect $u_{2}$ to some $u_{2,1}$ which is $C^{\infty}$ near $T_{1} \cup\left\{\partial T_{2} \cup \cdots \cup \partial T_{l}\right\}$. Along the homotopy the values of $u_{2}$ outside $T_{1}$ are preserved, so we make such homotopies successively on $T_{2}, \cdots, T_{l}$ and end up with some $v \in C^{\infty}\left(M, \mathbb{S}^{1}\right)$. Theorem 0.5 is established.

We now turn to the proof of Theorem 0.6. We first recall some notions already mentioned in the introduction. Denote by $[u]$ and $[u]_{p}$ the equivalence classes associated with $\sim$ and $\sim_{p}$. We have a well-defined map

$$
i_{p}:[u] \rightarrow[u]_{p}
$$

going from $C^{1}(M, N) / \sim$ to $W^{1, p}(M, N) / \sim_{p}$.

Recall

Definition 0.1. If $i_{p}$ is bijective, we say that $W^{1, p}(M, N)$ and $C^{0}(M, N)$ have the same topology.

With this definition we have

Theorem 0.6. For any $p \geq 2$ and any $M, W^{1, p}\left(M, \mathbb{S}^{1}\right)$ and $C^{0}\left(M, \mathbb{S}^{1}\right)$ have the same topology.

Proof. Let $n=\operatorname{dim} M$. If $n=2$, we know the result (Proposition 0.3). Also, the surjectivity of $i_{p}$ has been proved in Theorem 0.5 . So we only need to show that $i_{p}$ is injective in dimension $n \geq 3$.

Let $u, v \in C^{\infty}\left(M, \mathbb{S}^{1}\right)$ be such that, for some $p \geq 2$,

$$
[u]_{p}=[v]_{p}
$$

i.e. there exists $u^{t} \in C\left([0,1], W^{1, p}\left(M, \mathbb{S}^{1}\right)\right)$ such that $u^{0}=u$ and $u^{1}=v$. It is known that the connected components of $C^{0}\left(M, \mathbb{S}^{1}\right)$ and $\operatorname{Hom}\left(\pi_{1}(M), \pi_{1}\left(\mathbb{S}^{1}\right)\right)$ have a natural one-toone correspondence (see, e.g., Corollary 6.20, page 244, [Wh]). Here $\operatorname{Hom}\left(\pi_{1}(M), \pi_{1}\left(\mathbb{S}^{1}\right)\right.$ ) denotes the set of homomorphisms from $\pi_{1}(M)$ to $\pi_{1}\left(\mathbb{S}^{1}\right)$. So, we only need to show that

$$
u_{*}=v_{*},
$$

where $u_{*}$ and $v_{*}$ are the homomorphisms from $\pi_{1}(M)$ to $\pi_{1}\left(\mathbb{S}^{1}\right)$ induced respectively by $u$ and $v$. 
Let $\alpha \in C^{0}\left(\mathbb{S}^{1}, M\right)$; we can find $\beta \in C^{1}\left(\mathbb{S}^{1}, M\right)$ such that $\beta^{\prime} \neq 0$ and $\beta$ is path-connected to $\alpha$ in $C^{0}\left(\mathbb{S}^{1}, M\right)$. We only need to show that $u \circ \beta$ and $v \circ \beta$ are in the same path-connected component of $C^{0}\left(\mathbb{S}^{1}, \mathbb{S}^{1}\right)$. This amounts to verifying that

$$
\operatorname{deg}(u \circ \beta)=\operatorname{deg}(v \circ \beta)
$$

where deg denotes the Brouwer degree (the winding number in this case).

Let $B$ denote the unit ball in $\mathbb{R}^{n-1}$ centered at the origin and let

$$
\varphi: \mathbb{S}^{1} \times B \rightarrow M
$$

be a smooth immersion to a tubular neighborhood of $\beta\left(\mathbb{S}^{1}\right)$ such that $\varphi\left(\mathbb{S}^{1} \times\{0\}\right)$ is a "double" of $\beta\left(\mathbb{S}^{1}\right)$ (going around twice). This implies that $[\tilde{\beta}]=[\beta]^{2}$ in $\pi_{1}(M)$, where $\tilde{\beta}=\varphi(\cdot \times\{0\})$. Since $M$ is oriented, we can actually take $\varphi$ with $\tilde{\beta}=\beta$.

Clearly, $u^{t} \circ \varphi \in C\left([0,1], W^{1, p}\left(\mathbb{S}^{1} \times B, \mathbb{S}^{1}\right)\right)$. Since $p \geq 2=\operatorname{dim} \mathbb{S}^{1}+1$, a degree has been defined in [BLMN] for maps in $W^{1, p}\left(\mathbb{S}^{1} \times B, \mathbb{S}^{1}\right)$; moreover, this degree is invariant under homotopy in $W^{1, p}\left(\mathbb{S}^{1} \times B, \mathbb{S}^{1}\right)$. Therefore the degrees of $u \circ \varphi\left(=u^{0} \circ \varphi\right)$ and $v \circ \varphi$ $\left(=u^{1} \circ \varphi\right)$ are the same. This implies

$$
\operatorname{deg}(u \circ \tilde{\beta})=\operatorname{deg}(v \circ \tilde{\beta})
$$

from which (7.3) follows immediately. Thus we have shown (7.2) and Theorem 0.6 is established.

\section{Section 8. Some properties of $C T(M, N)$}

First recall some easy facts about " " and " p " which are proved in the Appendix.

Lemma 8.1. Let $u, v \in W^{1, p}(M, N) \cap C^{0}(M, N), 1 \leq p<\infty$, with $u \sim v$. Then $u \sim_{p} v$.

Warning: the converse is not true. However we have

Lemma 8.2. Let $u, v \in W^{1, p}(M, N) \cap C^{0}(M, N), p \geq \operatorname{dim} M$, with $u \sim_{p} v$.. Then $u \sim v$.

For $q \geq p$, we have a well-defined map

$$
i_{p, q}: W^{1, q}(M, N) / \sim_{q} \rightarrow W^{1, p}(M, N) / \sim_{p}
$$

Recall the following

Definition 0.2. Let $1<p<\infty$. We say that a change of topology occurs at $p$ if $\forall 0<$ $\epsilon<p-1, i_{p-\epsilon, p+\epsilon}$ is not bijective. Otherwise we say that there is no change of topology at p. We denote by $C T(M, N)$ the set of $p^{\prime} s$ where a change of topology occurs.

We now prove 
Proposition 0.4. $C T(M, N)$ is a compact subset of $[2, \operatorname{dim} M]$.

Proof. First observe that

$$
i_{p_{1}, p_{3}}=i_{p_{1}, p_{2}} \circ i_{p_{2}, p_{3}}, \quad \forall p_{1} \leq p_{2} \leq p_{3}
$$

Note that if $p>1$ is not in $C T$, then there exists $0<\epsilon<p-1$ such that $i_{p_{1}, p_{2}}$ is bijective for all $p-\epsilon<p_{1} \leq p_{2}<p+\epsilon$. Consequently, $C T$ is closed. By Theorem 0.2, for every $1 \leq p<2, W^{1, p}(M, N) / \sim_{p}$ consists of a single point; therefore

$$
C T(M, N) \cap[1,2)=\emptyset .
$$

On the other hand, it is clear that

$$
i_{p}=i_{p, q} \circ i_{q}, \quad \forall 1 \leq p \leq q<\infty
$$

Consequently, by Proposition $0.3, i_{p, q}$ is bijective for all $q \geq p \geq \operatorname{dim} M$, i.e.

$$
C T(M, N) \cap(\operatorname{dim} M, \infty)=\emptyset .
$$

An easy consequence of the definition of $C T$ is

Lemma 8.3. Let $1 \leq p \leq q<\infty$ be such that $[p, q] \cap C T(M, N)=\emptyset$. Then $i_{p, q}$ is bijective. Proof. For every $r \in[p, q]$, there exists $\epsilon=\epsilon(r)>0$ such that $i_{p_{1}, p_{2}}$ is bijective for $r-\epsilon<p_{1} \leq p_{2}<r+\epsilon$. Take a finite covering of $[p, q]$ by such intervals and apply (8.1).

Next we recall and prove

Proposition 0.5. If $C T(M, N)=\emptyset$ then $C^{0}(M, N)$ and $W^{1, p}(M, N)$ are path-connected for all $p \geq 1$.

Proof. Since $C T(M, N)=\emptyset$, it follows from Lemma 8.3 that

$$
i_{p, q} \text { is bijective } \quad \forall 1 \leq p \leq q<\infty \text {. }
$$

We know from Theorem 0.2 that $W^{1, p}(M, N)$ is path-connected for $1 \leq p<2$. It follows from (8.3) that $W^{1, q}(M, N)$ is also path-connected for $2 \leq q<\infty$. Choosing $q>\operatorname{dim} M$, we deduce, using Proposition 0.3, that $C^{0}(M, N)$ is also path-connected.

We now present the proofs of assertions (0.1)-(0.6) in the Introduction.

Proof of (0.1). This is a consequence of the fact that $W^{1, p}\left(B_{1}^{n}, N\right)$ is path-connected for all $1 \leq p<\infty$; see Proposition 1.6.

Proof of (0.2). This is a consequence of Proposition 0.3 and Proposition 0.1. 
Proof of (0.4). This is a consequence of Theorem 0.2 and Theorem 0.6.

Proof of (0.5). This is a consequence of Theorem 0.3 (or rather its equivalent form Theorem $0.3^{\prime}$ at the beginning of Section 5).

Proof of (0.6). It follows from Theorem 0.3 that $W^{1, p}\left(\mathbb{S}^{n} \times \Lambda, \mathbb{S}^{n}\right)$ is path-connected for all $1 \leq p<n+1$. On the other hand, as explained in Remark $0.1, W^{1, p}\left(\mathbb{S}^{n} \times \Lambda, \mathbb{S}^{n}\right)$ is not path-connected for all $p \geq n+1$.

From the above examples the reader might be tempted to think that $C T(M, N)$ is either empty or consists of a single point. As we have mentioned in the Introduction (see Open Problem), we believe that $C T(M, N)$ has usually more than one point. Here is a simple example where $C T$ contains exactly two points.

\section{Proposition 8.1.}

$$
C T\left(\mathbb{S}^{1} \times \mathbb{S}^{2}, \mathbb{S}^{1} \times \mathbb{S}^{2}\right)=\{2,3\}
$$

Moreover, let $u=\left(u_{1}, u_{2}\right), v=\left(v_{1}, v_{2}\right) \in W^{1, p}\left(\mathbb{S}^{1} \times \mathbb{S}^{2}, \mathbb{S}^{1} \times \mathbb{S}^{2}\right)$, then

a) For $p<2, u \sim_{p} v$,

b) For $2 \leq p<3, u \sim_{p} v$ if and only if

$$
\operatorname{deg}\left(u_{1}(\cdot, y)\right)=\operatorname{deg}\left(v_{1}(\cdot, y)\right), \quad \text { a.e. } y \in \mathbb{S}^{2},
$$

c) For $p \geq 3, W^{1, p}\left(\mathbb{S}^{1} \times \mathbb{S}^{2}, \mathbb{S}^{1} \times \mathbb{S}^{2}\right)$ and $C^{0}\left(\mathbb{S}^{1} \times \mathbb{S}^{2}, \mathbb{S}^{1} \times \mathbb{S}^{2}\right)$ have the same topology.

Proof. We first show that

$$
C T\left(\mathbb{S}^{1} \times \mathbb{S}^{2}, \mathbb{S}^{1}\right)=\{2\} .
$$

It follows from Theorem 0.2 that $W^{1, p}\left(\mathbb{S}^{1} \times \mathbb{S}^{2}, \mathbb{S}^{1}\right)$ is path-connected for all $1 \leq p<2$. On the other hand, it follows from Theorem 0.6 that $i_{p}$ is bijective for all $p \geq 2$. Therefore, since

$$
i_{q}=i_{p, q} \circ i_{p} \quad \forall p \leq q
$$

$i_{p, q}$ is bijective for all $2 \leq p \leq q$. This proves (8.6). We next show that

$$
C T\left(\mathbb{S}^{1} \times \mathbb{S}^{2}, \mathbb{S}^{2}\right)=\{3\} .
$$

It follows from Theorem 0.3 that $W^{1, p}\left(\mathbb{S}^{1} \times \mathbb{S}^{2}, \mathbb{S}^{2}\right)$ is path-connected for all $p<3$. On the other hand, by the Sobolev embedding theorem, $W^{1, p}\left(\mathbb{S}^{1} \times \mathbb{S}^{2}, \mathbb{S}^{2}\right)$ and $C^{0}\left(\mathbb{S}^{1} \times \mathbb{S}^{2}, \mathbb{S}^{2}\right)$ have the same topology for all $p>3$. This proves (8.7).

It is easy to see that $W^{1, p}\left(M, N_{1} \times N_{2}\right)=W^{1, p}\left(M, N_{1}\right) \times W^{1, p}\left(M, N_{2}\right)$, and $u=$ $\left(u_{1}, u_{2}\right) \sim_{p} v=\left(v_{1}, v_{2}\right)$ in $W^{1, p}\left(M, N_{1} \times N_{2}\right)$ if and only if $u_{1} \sim_{p} v_{1}$ in $W^{1, p}\left(M, N_{1}\right)$ and $u_{2} \sim_{p} v_{2}$ in $W^{1, p}\left(M, N_{2}\right)$. It follows that

$$
C T\left(M, N_{1} \times N_{2}\right)=C T\left(M, N_{1}\right) \cup C T\left(M, N_{2}\right) .
$$

(8.4) follows from (8.6), (8.7) and the above formula.

Part a) follows from Theorem 0.2. For $2 \leq p<3$, it follows from Theorem 0.6 that $u_{1} \sim_{p} v_{1}$ if and only if (8.5) holds, and, by Theorem 0.3, $u_{2} \sim_{p} v_{2}$. Part b) follows immediately. Part c) follows from Proposition 0.3. 


\section{APPENDiX}

In this Appendix we present, for the convenience of the reader, some results which are known to the experts.

Let $M$ and $N$ be compact, connected, oriented, smooth Riemannian manifolds with or without boundary. We assume that $N$ is smoothly embedded in some Euclidean space $\mathbb{R}^{K}$, so that, for some $\delta>0$, the projection $P$ of the $\delta$-neighborhood of $N$ (in $\mathbb{R}^{K}$ ) onto $N$ is well-defined and smooth. Recall that

$$
W^{1, p}(M, N)=\left\{u \in W^{1, p}\left(M, \mathbb{R}^{K}\right) ; u(x) \in N \text { a.e. }\right\}, \text { with } 1 \leq p<\infty
$$

Remark A.1. If $N$ is not compact we need a further assumption. Namely, we assume that $N$ is smoothly embedded in some Euclidean space $\mathbb{R}^{K}$, and, for some $\delta>0$, the projection $P$ of the $\delta$-neighborhood of $N$ (in $\mathbb{R}^{K}$ ) onto $N$ is well-defined and the gradient of $P$ (as a map from the $\delta$-neighborhood of $N$ to $\mathbb{R}^{K}$ ) is bounded in the $\delta$-neighborhood.

We first have

Proposition A.1. For $1 \leq p<\infty$, let $u, v \in W^{1, p}(M, N) \cap C^{0}(M, N)$ satisfying $u \sim v$. Then $u \sim_{p} v$.

Remark A.2. It follows from Proposition A.1 that $i_{p}$ is well defined.

Next we have

Proposition A.2. Let $u \in W^{1, p}(M, N)$ with $p \geq \operatorname{dim} M$. Then there exists $u^{t} \in C\left([0,1], W^{1, p}(M, N)\right)$ such that $u^{0}=u$ and $u^{t} \in C^{\infty}(M, N)$ for all $0<t \leq 1$.

Remark A.3. It follows from Proposition A.2 that Conjecture 1 holds for $p \geq \operatorname{dim} M$.

We also have

Proposition A.3. For $p \geq \operatorname{dim} M$, let $u, v \in W^{1, p}(M, N) \cap C^{0}(M, N)$ satisfy $u \sim_{p} v$. Then $u \sim v$.

Remark A.4. It follows from Proposition A.3 that $i_{p}: C^{1}(M, N) / \sim \rightarrow W^{1, p}(M, N) / \sim_{p}$ is injective for $p \geq \operatorname{dim} M$.

Remark A.5. Proposition 0.3 in the Introduction follows from Remark A.2 and Remark A.4.

The proofs of Propositions A.1-A.3 rely on some standard smoothing arguments. For the proofs of Proposition A.2-A.3 in the case $p=\operatorname{dim} M$, we also need the Poincaré inequality.

For simplicity we only consider the case where $\partial M=\emptyset$. We introduce a family of mollifiers on $M$ as follows. Let $\rho \in C^{\infty}\left(\mathbb{R}^{n}\right), \rho$ radially symmetric, $0 \leq \rho \leq 1$, supp $\rho \subset B_{1}$, $\int_{\mathbb{R}^{n}} \rho=1$. For $0<\epsilon<\epsilon_{1}\left(\epsilon_{1}\right.$ being the injectivity radius of $\left.M\right)$ and $x \in M$, the function

$$
\bar{\rho}_{\epsilon, x}(y)=\frac{1}{\epsilon^{n}} \rho\left(\exp _{x}^{-1}(y) / \epsilon\right)
$$


may not have total integral equal to 1 , so we normalize it by setting

$$
\rho_{\epsilon, x}(y)=\bar{\rho}_{\epsilon, x}(y) / \int_{M} \bar{\rho}_{\epsilon, x} .
$$

For $u \in W^{1, p}(M, N)$, let

$$
u_{\epsilon}(x)=\int_{M} \rho_{\epsilon, x} u
$$

It is easy to establish

Lemma A.1. Given $\delta>0$ and $u \in C^{0}(M, N)$, there exists $\epsilon_{2} \in\left(0, \epsilon_{1}\right)$, depending only on $\delta, M, N, \rho$, and the modulus of continuity of $u$, such that

$$
\left|u_{\epsilon}(x)-u(x)\right| \leq \delta, \quad \forall 0<\epsilon \leq \epsilon_{2}, \text { and } \forall x \in M .
$$

Consequently,

$$
\operatorname{dist}\left(u_{\epsilon}(x), N\right) \leq \delta, \quad \forall 0<\epsilon \leq \epsilon_{2}, \text { and } \forall x \in M
$$

Proof of Proposition A.1. Let $u^{t} \in C\left([0,1], C^{0}(M, N)\right)$ be such that $u^{0}=u$ and $u^{1}=v$, and let $P$ be the projection of some $\delta$-neighborhood of $N$ onto $N$ described at the beginning of the Appendix. Since the family has a uniform modulus of continuity, the $\epsilon_{2}$ in Lemma A. 1 can be taken uniform in $0 \leq t \leq 1$.

Define

$$
U^{t}= \begin{cases}P\left(u_{3 t \epsilon_{2}}^{0}\right) & 0 \leq t \leq 1 / 3 \\ P\left(u_{\epsilon_{2}}^{3 t-1}\right) & 1 / 3<t<2 / 3 \\ P\left(u_{(3-3 t) \epsilon_{2}}^{1}\right) & 2 / 3 \leq t \leq 1\end{cases}
$$

Clearly $U^{t} \in C\left([0,1], W^{1, p}(M, N)\right), U^{0}=u$, and $U^{1}=v$.

Proof of Proposition A.2 when $p>\operatorname{dim} M$. It follows from the Sobolev embedding theorem that $u \in C^{0}(M, N)$. Let $P$ be the projection of some $\delta$-neighborhood of $N$ onto $N$ described at the beginning of the Appendix, and let $\epsilon_{2}$ be the number given in Lemma A.1. Define

$$
u^{t}=P\left(u_{t \epsilon_{2}}\right) \text {. }
$$

Clearly this is a homotopy with the desired properties.

The proof of Proposition A.2 when $p=\operatorname{dim} M$ relies on the following Poincaré inequality: For $p=\operatorname{dim} M, 0<\epsilon \leq \epsilon_{1}, x \in M, u \in W^{1, p}(M, N)$, we have

$$
\frac{1}{\epsilon^{p}} \int_{B_{\epsilon}(x)}\left|u-u_{\epsilon}(x)\right|^{p} \leq C \int_{B_{\epsilon}(x)}|\nabla u|^{p},
$$

where $B_{\epsilon}(x)$ denotes the $\epsilon$-geodesic ball centered at $x$, the integration and the gradient $\nabla$ is with respect to the Riemannian metric on $M$, and the constant $C$ depends only on the manifolds $M$ and $N$. Consequently we have 
Lemma A.2. For $u \in W^{1, p}(M, N), p=\operatorname{dim} M, 0<\epsilon<\epsilon_{1}$, we have

$$
\sup _{x \in M} \operatorname{dist}\left(u_{\epsilon}(x), N\right)^{p} \leq C \sup _{x \in M} \int_{B_{\epsilon}(x)}|\nabla u|^{p},
$$

where $C=C(M, N)$.

Proof of Proposition A.2 when $p=\operatorname{dim} M$. Because of Lemma A.2, $\epsilon_{2}$ can be found so that (A.2) is satisfied. The rest is identical to the proof for the case $p>\operatorname{dim} M$.

Proof of Proposition A.3. Let $u^{t} \in C\left([0,1], W^{1, p}(M, N)\right)$ such that $u^{0}=u$ and $u^{1}=v$. If $p>\operatorname{dim} M$, it follows from the Sobolev embedding theorem that $W^{1, p}(M, N) \subset C^{0}(M, N)$. So $u^{t} \in C\left([0,1], C^{0}(M, N)\right)$, and $u \sim v$.

For $p=\operatorname{dim} M$, let $P$ be the projection of some $\delta$-neighborhood of $N$ onto $N$ described at the beginning of the Appendix. We observe that $\left\{u^{t}\right\}_{0 \leq t \leq 1}$ is a compact subset of $W^{1, p}(M, N)$, so, in view of Lemma A.2, there exists $\epsilon_{2}>0$ such that

$$
\operatorname{dist}\left(u_{\epsilon}(x), N\right) \leq \delta, \quad \forall 0 \leq t \leq 1,0<\epsilon \leq \epsilon_{2}, x \in M .
$$

Therefore the homotopy $\left\{U^{t}\right\}$ in the proof of Proposition A.1 is well-defined and has the desired properties.

To complete the Appendix, we present the following propositions which are used in the proofs of Proposition 6.1 and Theorem 0.5 respectively.

Proposition A.4. Let $p \geq 1$, and let $O$ be an (relative) open subset of $M$. Then for every $u \in W^{1, p}(M, N) \cap C^{0}(O)$, there exists $u^{t} \in C\left([0,1], W^{1, p}(M, N)\right)$ such that

$$
\begin{aligned}
& u^{t} \in C^{0}(O), \quad \forall 0 \leq t \leq 1, \\
& u^{0}=u, \\
& u^{t} \in C^{\infty}(O), \quad \forall 0<t \leq 1 .
\end{aligned}
$$

Proof. For simplicity we only consider the case where $\partial M=\emptyset$. We adapt the classical argument of Meyers-Serrin [MS]. Let $O_{j}, j=1,2, \cdots$, be a sequence of open subsets strictly contained in $O$ satisfying $O_{j} \subset \subset O_{j+1}$ and $O=\cup O_{j}$, and let $\left\{\psi_{j}\right\}_{j \geq 0}$ be a smooth partition of unity subordinate to the covering $\left\{O_{j+1} \backslash \overline{O_{j-1}}\right\}_{j \geq 0}\left(O_{0}\right.$ and $O_{-1}$ being defined as empty set). We choose $\epsilon_{j}, j=1,2, \cdots$, satisfying

$$
\epsilon_{j} \leq \operatorname{dist}\left(O_{j}, \partial O_{j+1}\right), \quad j \geq 1,
$$

$$
\left\|\left(\psi_{j} u\right)_{t \epsilon_{j}}-\left(\psi_{j} u\right)\right\|_{C^{0}(O)}+\left\|\left(\psi_{j} u\right)_{t \epsilon_{j}}-\left(\psi_{j} u\right)\right\|_{W^{1, p}(O)} \leq \frac{\delta}{2^{j}}, \quad \forall 0<t \leq 1,
$$


where $\left(\psi_{j} u\right)_{t \epsilon_{j}}$ is defined as in (A.1).

Set

$$
\begin{aligned}
& v^{t}=\sum_{j}\left(\psi_{j} u\right)_{t \epsilon_{j}}, \quad 0<t \leq 1, \\
& v^{0}=u .
\end{aligned}
$$

It follows from (A.4) that

$$
\left\|v^{t}-u\right\|_{C^{0}(O)} \leq \sum_{j}\left\|\left(\psi_{j} u\right)_{t \epsilon_{j}}-\left(\psi_{j} u\right)\right\|_{C^{0}} \leq \delta
$$

and

$$
\left\|v^{t}-u\right\|_{W^{1, p}(O)} \leq \sum_{j}\left\|\left(\psi_{j} u\right)_{t \epsilon_{j}}-\left(\psi_{j} u\right)\right\|_{W^{1, p}} \leq \delta .
$$

For fixed $j$,

$$
\lim _{t \rightarrow 0}\left(\left\|\left(\psi_{j} u\right)_{t \epsilon_{j}}-\left(\psi_{j} u\right)\right\|_{C^{0}(O)}+\left\|\left(\psi_{j} u\right)_{t \epsilon_{j}}-\left(\psi_{j} u\right)\right\|_{W^{1, p}(O)}\right)=0 .
$$

So, by the Lebesgue dominated convergence theorem (using (A.4)), we have

$$
\lim _{t \rightarrow 0}\left(\left\|v^{t}-u\right\|_{C^{0}(O)}+\left\|v^{t}-u\right\|_{W^{1, p}(O)}\right)=0 .
$$

Similarly, for every $0<s \leq 1$,

$$
\lim _{t \rightarrow s}\left(\left\|v^{t}-v^{s}\right\|_{C^{0}(O)}+\left\|v^{t}-v^{s}\right\|_{W^{1, p}(O)}\right)=0 .
$$

It follows from (A.5), (A.7) and (A.8) that

$$
u^{t}=P\left(v^{t}\right), \quad 0 \leq t \leq 1,
$$

is well-defined and satisfies the desired properties.

Finally, a variant of Proposition A.4. Let $O$ be an open subset of $M$ and $K$ be a compact subset of $O$. For $\epsilon>0$, set $K_{\epsilon}=\{x \in M ; \operatorname{dist}(x, K) \leq \epsilon\}$. 
Proposition A.5. Let $1 \leq p<\infty$, and let $K \subset O \subset M$ be as above. Then for every $u \in W^{1, p}(M, N) \cap C^{0}(O)$, there exist $\epsilon>0$ and $u^{t} \in C\left([0,1], W^{1, p}(M, N) \cap C^{0}(O)\right)$ such that

$$
\begin{aligned}
& u^{t} \in C^{0}(O) \quad \forall 0 \leq t \leq 1, \\
& u^{t}(x)=u(x) \quad \forall 0 \leq t \leq 1, x \in M \backslash K_{2 \epsilon}, \\
& u^{0}=u \quad \text { and } \quad u^{t} \in C^{\infty}\left(K_{\epsilon}\right) \quad \forall 0<t \leq 1 .
\end{aligned}
$$

Proof. For $\epsilon>0$ with $K_{3 \epsilon} \subset O$, let $\eta \in C^{\infty}(M)$ be a cut-off function with

$$
\eta= \begin{cases}1 & x \in K_{2 \epsilon}, \\ 0 & x \in M \backslash K_{3 \epsilon},\end{cases}
$$

and let

$$
v^{t}=\int_{M} \rho_{t \epsilon, x} u, \quad 0 \leq t \leq 1,
$$

where $\rho_{t \epsilon, x}$ is defined above.

Consider

$$
u^{t}=P\left((1-\eta) u+\eta v^{t}\right), \quad 0 \leq t \leq 1,
$$

where $P$ is the smooth projection of the $\delta$-neighborhood of $N$ onto $N$. It is clear that, for small $\epsilon, u^{t}$ is a desired homotopy.

\section{References}

[B] F. Bethuel, The approximation problem for Sobolev maps between two manifolds, Acta Math. 167 (1991), 153-206.

[BZ] F. Bethuel and X. Zheng, Density of smooth functions between two manifolds in Sobolev spaces, J. Funct. Anal. 80 (1988), 60-75.

[BBM] J. Bourgain, H. Brezis and P. Mironescu, Lifting in Sobolev spaces, J. d'Analyse, to appear.

[BLMN] H. Brezis, Y.Y. Li, P. Mironescu and L. Nirenberg, Degree and Sobolev spaces, Topological Methods in Nonlinear Analysis, 13 (1999), 181-190.

[BN] H. Brezis and L. Nirenberg, Degree theory and BMO, Part I: Compact manifolds without boundaries, Selecta Math. 1 (1995), 197-263; Part II: Compact manifolds with boundaries, Selecta Math. 2 (1996), 309-368.

[MS] N.G. Meyers and J. Serrin, $H=W$, Proc. Nat. Acad. Sci. U.S.A. 51 (1964), 1055-1056. 
[RS] J. Rubinstein and P. Sternberg, Homotopy classification of minimizers of the Ginzburg-Landau energy and the existence of permanent currents, Comm. Math. Phys. 179 (1996), 257-263.

[SU] R. Schoen and K. Uhlenbeck, Boundary regularity and the Dirichlet problem for harmonic maps, J. Diff. Geom. 18 (1983), 253-268.

[S] E.M. Stein, Singular integrals and differentiability properties of functions, Princeton University Press, Princeton, New Jersey, 1970.

[W1] B. White, Infima of energy functionals in homotopy classes of mappings, J. Diff. Geom. 23 (1986), 127-142

[W2] B. White, Homotopy classes in Sobolev spaces and the existence of energy minimizing maps, Acta Math. 160 (1988), 1-17.

[Wh] G.W. Whitehead, Elements of homotopy theory, Springer-Verlag, New York, 1978.

(1) Analyse NumÉrique

Université P. et M. Curie, B.C. 187

4 PL. JUSSIEU

75252 PARIS CEDEX 05

E-mail address: brezis@ccr.jussieu.fr

(2) Rutgers University

Dept. of Math., Hill Center, Busch Campus

110 Frelinghuysen Rd, Piscataway, NJ 08854

E-mail address: brezis@math.rutgers.edu; yyli@math.rutgers.edu 